\title{
Stable Sunspot Solutions in Models with Predetermined Variables*
}

\author{
George W. Evans \\ University of Oregon
}

\author{
Bruce McGough \\ Oregon State University
}

April 17, 2002

\begin{abstract}
We consider a linear stochastic univariate rational expectations model, with a predetermined variable, and provide alternative representations of SSEs (stationary sunspot equilibria). For a strict subset of the parameter space there exist SSEs that are locally stable under least squares learning provided agents use a common factor representation for their estimated law of motion. These results indicate that for some parameter regions SSEs are more likely to arise under private agent learning than previously recognized.
\end{abstract}

\section{Introduction}

The possibility of expectations driven fluctuations in economic activity, emphasized by (Keynes 1936), received new support with the work of (Shell 1977), (Azariadis 1981), (Cass and Shell 1983) and (Guesnerie 1986), in which it was shown that some market clearing models could have "self-fulfilling" solutions, under rational expectations, driven by extraneous stochastic processes known as "sunspots." Although sunspot equilibria were first shown to exist in simple stylized models, such as the Overlapping Generations model of money $^{1}$, the line of research initiated by (Benhabib and Farmer 1994),

${ }^{*}$ Financial support from the National Science Foundation is gratefully acknowledged.

${ }^{1}$ See the extensive survey in (Guesnerie and Woodford 1992). 
(Farmer and Guo 1994) and (Farmer 1999) has shown the possibility of sunspot equilibria in versions of Real Business Cycle models with monopolistic competition or externalities.

A question that has accompanied the growth of this literature concerns the attainability of such equilibria. That sunspot solutions could be stable under adaptive learning was demonstrated for the basic Overlapping Generations model by (Woodford 1990), and conditions for local stability under adaptive learning were provided in (Evans and Honkapohja 1994b) and (Evans and Honkapohja 2002a) for one-step forward looking univariate nonlinear models. The solutions considered in these papers take the form of a dependence on extraneous finite state Markov processes. This type of solution is prominent in the theoretical literature, e.g. see (Chiappori and Guesnerie 1989), but plays a relatively minor role in applied macroeconomic research.

A different class of sunspot solutions, taking an autoregressive-moving average (ARMA) form, has also been discussed in the literature, e.g. (McCallum 1983), (Farmer 1999), and Part III of (Evans and Honkapohja 2001). In these formulations the extraneous sunspot variable is an exogenous white noise process. (Evans and Honkapohja 1994a) and (Evans and Honkapohja 2001) discuss the stability under learning, for linear models with predetermined variables, of sunspot solutions taking this ARMA form, which is the type of solution more commonly considered in the business cycle literature.

The current paper reexamines these issues in the context of a linear expectations model with a predetermined variable. Our major contribution is to show that a subset of ARMA-type stationary sunspot equilibria, which would previously have been viewed as unstable under learning, are stable when agents condition their forecasts on an appropriate exogenous variable. This finding substantially increases the set of stationary sunspot equilibria that are plausible solutions, in the sense of being locally learnable, and it includes types of solutions often emphasized in applied work.

We emphasize throughout that rational expectations equilibria have alternative representations, and we show how stability under learning depends in part on the representation estimated by agents and used by them to make forecasts. Although the "general form" ARMA representations that depend on serially uncorrelated sunspot variables, are not stable under learning, we find that alternative "common factor" representations of these solutions are in some cases stable. One way to view our results is that they show how stability under learning of sunspot equilibria can depend on the time-series 
structure of the observed sunspot variable. For part of the parameter space, exogenous autoregressive variables with an appropriate autocorrelation coefficient (a "resonant frequency") can induce sunspot equilibria that are stable under learning.

We illustrate these results using Sargent's extension of the Lucas-Prescott model of investment under uncertainty to incorporate tax distortions and externalities. Stationary sunspot equilibria exist for some cases in which taxrates and/or externalities are large. In part of the "indeterminacy region," although sunspot equilibria exist, they are never stable under learning. However, in other regions of the parameter space these stationary sunspot equilibria are stable under least squares learning provided agents condition on a sunspot variable with the required time-series properties.

\section{The Framework}

We consider the following univariate model:

$$
y_{t}=\alpha+\beta E_{t}^{*} y_{t+1}+\delta y_{t-1}+v_{t},
$$

where $v_{t}$ is a white noise exogenous process and $E_{t}^{*} y_{t+1}$ denotes the expectations formed by agents at the start of period $t$. Under the rational expectations assumption $E_{t}^{*} y_{t+1}=E_{t} y_{t+1}$, the mathematical expectation of $y_{t+1}$ conditional on information available at $t$.

Clearly we want to impose that $\beta \neq 0$ and $\delta \neq 0$ and we will also assume that $\beta+\delta \neq 1$. There is then a unique nonstochastic steady state given by $\bar{y}=(1-\beta-\delta)^{-1} \alpha$. Hereafter we let $y_{t}, y_{t-1}, E_{t}^{*} y_{t+1}$, etc., denote deviations from $\bar{y}$, and we can therefore rewrite the model as

$$
y_{t}=\beta E_{t}^{*} y_{t+1}+\delta y_{t-1}+v_{t} .
$$

Our focus will be on stochastic versions of the model in which $v_{t}$ is nontrivial, but we will also at times need to refer to the nonstochastic model

$$
y_{t}=\beta E_{t}^{*} y_{t+1}+\delta y_{t-1},
$$

which appears frequently in the literature. Note that, even though there are no "intrinsic" shocks in (2), the nonstochastic model can have stochastic solutions under rational expectations. ${ }^{2}$ In the stochastic case solutions will

\footnotetext{
${ }^{2}$ The set of solutions to the model without predetermined variables was discussed in detail by (Gourieroux, Laffont, and Monfort 1982).
} 
depend on $v_{t}$ and may also depend on other extraneous stochastic processes, usually called "sunspots." In this paper we begin by characterizing the solutions to (1), i.e. the rational expectations solutions to the model. We then relax the assumption that expectations are given by true conditional expectations and consider the system under learning.

A rational expectations equilibrium (REE) is a stochastic process $y_{t}$ which satisfies (1) or (2) under the assumption that $E_{t}^{*} y_{t+1}=E_{t} y_{t+1}$. The model (1) can be regarded as holding for all integers $t$, in which case REE solutions $y_{t}$ are doubly infinite stochastic processes. Alternatively the model can be assumed to hold for all integers $t \geq t_{0}$, where without loss of generality we set $t_{0}=0$. In the latter case REE are solutions that begin at time $t=0$ with some initial distribution $y_{0}$, which for convenience we take to be a nonstochastic initial condition $\breve{y}_{0}$. In this case we say that the REE $y_{t}$ is an initialized stochastic process. We will focus on the doubly infinite case, but occasionally will need to explicitly discuss initialized models.

We restrict attention to solutions that are nonexplosive in the sense that, for each $t, E_{t}\left|y_{t+s}\right|$ is uniformly bounded over $s$. The applied literature frequently focuses on solutions that are covariance stationary, in the case of doubly infinite processes, or asymptotically covariance stationary, in the case of initialized processes. It can be shown that if a process is covariance stationary, or asymptotically covariance stationary, then it is uniformly bounded in expectation, and that the latter condition in turn implies that it is conditionally uniformly bounded in expectation. A statement and proof of these results is provided in the Appendix.

The nonstochastic model always has the stationary solution $y_{t}=0$, but whether or not (1) has a nonexplosive stationary solution depends on the roots of the associated quadratic $\beta a^{2}-a+\delta$,

$$
a_{1}=\frac{1-\sqrt{1-4 \beta \delta}}{2 \beta} \text { and } a_{2}=\frac{1+\sqrt{1-4 \beta \delta}}{2 \beta} .
$$

Throughout the paper, for convenience, we will assume that the two roots are distinct and that neither lies on the unit circle. If both roots are greater than one in norm ${ }^{3}$ then, as we show below, there are no nonexplosive solutions to the model. If the quadratic $\beta a^{2}-a+\delta$ has exactly one real root $a_{i}$ with

\footnotetext{
${ }^{3}$ i.e., have absolute value greater than one if real or complex modulus greater than one if not real.
} 
norm less than one then there is a stationary solution given by

$$
z_{t}^{i}=\left(1-a_{i} L\right)^{-1}\left(1-\beta a_{i}\right)^{-1} v_{t}=\left(1-\beta a_{i}\right)^{-1} \sum_{j=0}^{\infty} a_{i}^{j} v_{t-j}
$$

Here $L$ is the lag operator defined by $L y_{t}=y_{t-1}$ and (3) explicitly gives the (absolutely summable) moving average representation of the solution $z_{t}^{i}$. It is easily verified that $z_{t}^{i}$ solves (1) and in the next section we show that it is the unique nonexplosive solution in this case. This solution, of course, has an $\mathrm{AR}(1)$, i.e. first order autoregressive, representation, which can be written as

$$
\begin{aligned}
z_{t}^{i} & =a_{i} z_{t-1}^{i}+\left(1-\beta a_{i}\right)^{-1} v_{t}, \text { or equivalently } \\
z_{t}^{i} & =a_{i} z_{t-1}^{i}+\left(\beta a_{j}\right)^{-1} v_{t}, \text { for } j \neq i
\end{aligned}
$$

where the second line uses $\beta\left(a_{1}+a_{2}\right)=1$.

If both roots of the associated quadratic are less than one in norm then there is a stationary solution given by

$$
\hat{z}_{t}=\left(1-\beta^{-1} L+\beta^{-1} \delta L^{2}\right)^{-1}\left(1-\beta^{-1} L\right) v_{t},
$$

as can again be directly verified. Note that this can also be written as the $\operatorname{ARMA}(2,1)$ process $\hat{z}_{t}=\beta^{-1} \hat{z}_{t-1}-\beta^{-1} \delta \hat{z}_{t-2}+v_{t}-\beta^{-1} v_{t-1}$. If the roots are real there also exist two solutions of the form (3), or equivalently (4), in which $a_{i}$ is chosen to be either of the two roots. These solutions are often referred to as the MSV (minimal state variable) solutions, following (McCallum 1983). ${ }^{4}$ However, if both roots are less than one in norm there are other stationary solutions as well, and the complete class of solutions can be described in various ways. It will be useful to note that the set of solutions to (1) is given by the sum of any particular solution plus the set of solutions to the homogeneous equation (2). Thus, $y_{t}=z_{t}^{i}+\zeta_{t}$ is a solution of model (1) if and only if the stochastic process $\zeta_{t}$ satisfies

$$
\zeta_{t}=\beta E_{t} \zeta_{t+1}+\delta \zeta_{t-1}
$$

\footnotetext{
${ }^{4}$ In fact (McCallum 1983) recommends choosing one of these two solutions based on a subsidiary selection principle, whereas we will focus on local stability under learning of alternative solutions.
} 
(If the roots are nonreal then the set of solutions can be written $\left.y_{t}=\hat{z}_{t}+\zeta_{t}\right) .^{5}$ When $\zeta_{t}$ is stationary and generated by an extraneous exogenous process, the solution is called a stationary sunspot equilibrium (SSE).

In the next section we provide a characterization of the full set of solutions, when multiple equilibria exist, in terms of martingale difference sequences. Although these and related results on regularity and determinacy are not new, they provide the starting point for our analysis of the stability of solutions under learning.

\section{Indeterminacy and Existence of SSEs}

Throughout this Section we maintain the rational expectations assumption. An issue usually discussed in the context of the nonstochastic model (2) is whether a solution is "locally unique" or "determinate." For this concept we consider initialized processes. Suppose that an initial condition $y_{0}=$ $\breve{y}_{0}$ is given and that (2) holds for $t=1,2,3, \ldots$ Consider first perfect foresight solutions, which satisfy $y_{t+1}=\beta^{-1} y_{t}-\beta^{-1} \delta y_{t-1}$, for $t=1,2,3, \ldots$. If the roots $a_{1}, a_{2}$ of the quadratic are real then solutions can be written $y_{t}=K_{1} a_{1}^{t}+K_{2} a_{2}^{t}$ for parameters $K_{1}, K_{2}$. The initial condition imposes the constraint $K_{1}+K_{2}=\breve{y}_{0}$. If $\left|a_{1}\right|,\left|a_{2}\right|>1$ then there is no nonexplosive solution unless $\breve{y}_{0}=0$. If $\left|a_{1}\right|<1<\left|a_{2}\right|$ then imposing nonexplosiveness leads to the additional condition $K_{2}=0$, and it follows that there is a unique nonexplosive solution. The model or solution in this case is said to be "determinate."

Finally, if $\left|a_{1}\right|,\left|a_{2}\right|<1$ then for every $K_{1}$ the sequence given for $t=$ $1,2,3, \ldots$ by $y_{t}=K_{1} a_{1}^{t}+\left(\breve{y}_{0}-K_{1}\right) a_{2}^{t}$ is a perfect foresight solution. In particular, if $y_{t}$ is the solution specified by $K_{1}$ and $y_{t}^{\prime}$ is the solution specified by $K_{1}^{\prime}$ then $\lim _{t \rightarrow \infty}\left|y_{t}-y_{t}^{\prime}\right|=0$ and $\sup _{t>1}\left|y_{t}-y_{t}^{\prime}\right|$ can be made arbitrarily small by choosing $K_{1}^{\prime}$ sufficiently close to $K_{1}$. We say that the model is "indeterminate" in the sense that for any perfect foresight solution there is another perfect foresight solution arbitrarily close to it. Analogous arguments can be made for the case of nonreal roots $b \pm c i$ since in this case perfect foresight solutions can be written $y_{t}=K r^{t} \cos (\omega t+\phi)$ where $r=\left(b^{2}+c^{2}\right)^{1 / 2}$ and $r \cos \omega=b$ : if $r>1$ there are no nonexplosive solution unless $\breve{y}_{0}=0$

\footnotetext{
${ }^{5}$ For $\zeta_{t}$ satisfying this equation and given any particular solution $z_{t}$, substitution of $y_{t}=z_{t}^{i}+\zeta_{t}$ into (1) verifies that $y_{t}$ is also a solution. Similarly if $y_{t}$ is a solution then letting $\zeta_{t}=y_{t}-z_{t}^{i}$ and substituting into (1) it follows that $\zeta_{t}=\beta E_{t} \zeta_{t+1}+\delta \zeta_{t-1}$.
} 
while if $r<1$ there are multiple nonexplosive solutions and the model is indeterminate. ${ }^{6}$

The closely related concepts of "regular" and "irregular" models are usually stated in terms of the matrix representation of the solutions. Writing the perfect foresight solutions as

$$
\begin{aligned}
{\left[\begin{array}{c}
y_{t+1} \\
y_{t}
\end{array}\right] } & =\left[\begin{array}{cc}
\beta^{-1} & -\beta^{-1} \delta \\
1 & 0
\end{array}\right]\left[\begin{array}{c}
y_{t} \\
y_{t-1}
\end{array}\right], \text { or } \\
x_{t+1} & =A x_{t},
\end{aligned}
$$

it is easily seen that the eigenvalues of $A$ are equal to the roots of the associated quadratic. If the number of roots of $A$ with norm less than one is equal to (more than, less than) the number of predetermined variables then the model is said to be regular (irregular, explosive). Since we here have one predetermined variable, it is immediate that regular models have determinate solutions while irregular models are indeterminate.

We now turn to the stochastic model (1) and restate and extend these results. Letting $\varepsilon_{t+1}=y_{t+1}-E_{t} y_{t+1}$, rational expectations implies that $E_{t} \varepsilon_{t+1}=0$. A stochastic process $\varepsilon_{t}$ that satisfies this property is known as a martingale difference sequence. It follows that a stochastic process $y_{t}$ that solves (1) must satisfy

$$
y_{t+1}=\beta^{-1} y_{t}-\beta^{-1} \delta y_{t-1}-\beta^{-1} v_{t}+\varepsilon_{t+1}
$$

for some martingale difference sequence $\varepsilon_{t+1}$. Conversely, for any arbitrary martingale difference sequence $\varepsilon_{t+1}$ this equation specifies a solution. ${ }^{7}$ It is convenient to rewrite the equation in matrix form as:

$$
\left[\begin{array}{c}
y_{t+1} \\
y_{t}
\end{array}\right]=\left[\begin{array}{cc}
\beta^{-1} & -\beta^{-1} \delta \\
1 & 0
\end{array}\right]\left[\begin{array}{c}
y_{t} \\
y_{t-1}
\end{array}\right]+\left[\begin{array}{c}
-\beta^{-1} \\
0
\end{array}\right] v_{t}+\left[\begin{array}{l}
1 \\
0
\end{array}\right] \varepsilon_{t+1} .
$$

As in the nonstochastic case we can distinguish between regular, irregular and explosive cases based on the number of roots of $A$ inside the unit circle.

Our definition of determinacy extends in a natural way to the stochastic case. Let $\left\{y_{t}\right\}_{t=0}^{\infty}$ be a solution to (1) subject to the initial condition $y_{0}=\breve{y}_{0}$.

\footnotetext{
${ }^{6}$ (Blanchard and Kahn 1980) showed, for a general class of linear models, that there is a unique nonexplosive solution in the "regular" case (see below).

${ }^{7}$ The approach of writing solutions to linear rational expectations models in terms of arbitrary martingale difference sequences was emphasized by (Broze, Gourieroux, and Szafarz 1990).
} 
The solution is said to be indeterminate if there exists a distinct solution $\left\{y_{t}^{\prime}\right\}_{t=0}^{\infty}$, meeting the initial condition, such that $\lim _{t \rightarrow \infty}\left|y_{t}-y_{t}^{\prime}\right|=0$ and $\sup _{t \geq 1}\left|y_{t}-y_{t}^{\prime}\right|$ can be made arbitrarily small, where equality here is taken to hold almost everywhere. Otherwise the solution is said to be determinate.

We have the following result:

Proposition 1 Let $a_{1}, a_{2}$ be the roots of the associated quadratic. (i) If $\left|a_{1}\right|,\left|a_{2}\right|>1$ then almost surely there exist no nonexplosive solutions to (1). (ii) In the regular case $\left|a_{1}\right|<1<\left|a_{2}\right|$ there is a unique nonexplosive solution to (1). This solution has the representation

$$
y_{t}=a_{1} y_{t-1}+\left(\beta a_{2}\right)^{-1} v_{t}
$$

which is stationary, in the doubly infinite case, and asymptotically stationary for the initialized model. It follows that there do not exist SSEs. (iii) In the irregular case $\left|a_{1}\right|,\left|a_{2}\right|<1$ SSEs exist. $y_{t}$ is a solution if and only if it can be written in the form (5) for some martingale difference sequence $\varepsilon_{t+1}$, and in this case every solution is indeterminate.

The region of the parameter space in which SSEs exist is given by the following:

Corollary 2 The model is irregular if and only if the parameters of the model are contained in one of the following two regions:

1. $\beta>\frac{1}{2}, \quad \delta>1-\beta, \quad \delta<\beta$

2. $\beta<-\frac{1}{2}, \quad \delta<-1-\beta, \quad \delta>\beta$

These results are proved in the Appendix.

It is also straightforward to compute that the regular case corresponds to the region $|\beta+\delta|<1$.

\section{Representations and T-maps}

Before considering the stability of REE under learning we must first distinguish between REEs and their representations. We begin by defining the notion of representations and then show how maps from the perceived to the actual law of motion, which we will call T-maps, can be used to characterize 
them. As will be shown later, alternative representations of an REE correspond to alternative econometric specifications that might naturally be used by an economic agent faced with the problem of how to forecast key variables required for decision making.

\subsection{Representations}

Recall the model (1) under the RE assumption:

$$
y_{t}=\beta E_{t} y_{t+1}+\delta y_{t-1}+v_{t},
$$

reproduced here for convenience. Recall that an REE is a stochastic process $y_{t}$ that satisfies this equation. A rational expectation equilibrium representation (REER) of a given REE is a linear recursion, a solution to which is the REE. We say the REE is represented by the REER. Notice that a given REE may have more than one representation: for example if a doubly infinite REE is represented by an $\mathrm{AR}(1)$ process then it is also represented by an $\operatorname{ARMA}(2,1)$; simply operate on each side of the $\mathrm{AR}(1)$ representation by $1-\eta L$ for any $\eta \in \mathbb{R}$. Also, a given REER may have many solutions and hence yield many REE's: for example, if an initialized model does not specify a full complement of initial conditions for the REER then the REER may have infinitely many solutions corresponding to the additional degrees of freedom in its initial conditions.

In the previous section we showed that, when the nonexplosiveness condition is imposed, in the regular case there is a unique REE. In this case the REE does not depend on sunspots and it has an $\mathrm{AR}(1)$ representation driven by the intrinsic shock. In contrast, in the irregular case there are multiple REE, including REE that depend on sunspots. We now consider in more detail representations of the REE in the irregular case. In the previous section we demonstrated the first result on representations of REEs, namely:

Proposition 3 In the irregular case, the process $y_{t}$ is an REE of (1) if and only if there is a martingale difference sequence $\varepsilon_{t}$ so that $y_{t}$ solves

$$
y_{t}=\beta^{-1} y_{t-1}-\beta^{-1} \delta y_{t-2}-\beta^{-1} v_{t-1}+\varepsilon_{t} .
$$

Representations of this form we call general form representations.

If we consider only the case with real roots and doubly infinite processes, we also have the following: 
Proposition 4 Consider the irregular case and assume that the roots of the associated quadratic are real. The process $y_{t}$ is an REE of (1) if and only if there is a martingale difference sequence $\varepsilon_{t}$ so that $y_{t}$ solves

$$
y_{t}=a_{i} y_{t-1}+\xi_{t}+\left(\beta a_{j}\right)^{-1} v_{t}
$$

where $a_{i}, i=1,2$ are the roots of the quadratic $\beta a^{2}-a+\delta=0, i \neq j$, and $\xi_{t}$ satisfies

$$
\xi_{t}=a_{j} \xi_{t-1}-\left(\beta a_{j}\right)^{-1} v_{t}+\varepsilon_{t} .
$$

Proof. By Proposition $3 y_{t}$ is an REE if and only if there exists a martingale difference sequence. $\varepsilon_{t}$ so that $y_{t}$ solves (7). Define the process $\xi_{t}$ by (9). Then $y_{t}$ is an REE if and only if

$$
\begin{aligned}
& \left(1-\beta^{-1} L+\beta^{-1} \delta L^{2}\right) y_{t}=\varepsilon_{t}-\beta^{-1} v_{t-1} \\
\Leftrightarrow & \left(1-a_{1} L\right)\left(1-a_{2} L\right) y_{t}=\xi_{t}-a_{j} \xi_{t-1}-\beta^{-1} v_{t-1}+\left(\beta a_{j}\right)^{-1} v_{t} \\
\Leftrightarrow & \left(1-a_{1} L\right)\left(1-a_{2} L\right) y_{t}=\left(1-a_{j} L\right) \xi_{t}+\left(\beta a_{j}\right)^{-1}\left(1-a_{j} L\right) v_{t} .
\end{aligned}
$$

Canceling the appropriate lag polynomial yields the result.

We will refer to representations of this form as common factor representations (CF representations) since they are obtained from lag polynomials with a common factor. ${ }^{8}$ These propositions tell us that each doubly infinite REE can be represented in general form and (in two ways) as a common factor. Several additional remarks are in order.

Remark 1: We can equivalently state that $y_{t}$ is an REE if and only if there is a martingale difference sequence $\tilde{\varepsilon}_{t}$ so that $y_{t}$ solves (8) and $\xi_{t}$ satisfies

$$
\xi_{t}=a_{j} \xi_{t-1}+\tilde{\varepsilon}_{t}
$$

This is immediate since $\varepsilon_{t}=\tilde{\varepsilon}_{t}+\left(\beta a_{j}\right)^{-1} v_{t}$ defines a martingale difference sequence.

Remark 2: Each of the two MSV solutions $y_{t}=a_{i} y_{t-1}+\left(\beta a_{j}\right)^{-1} v_{t}$ also has two CF representations. One representation is $y_{t}=a_{i} y_{t-1}+\xi_{t}+\left(\beta a_{j}\right)^{-1} v_{t}$

${ }^{8}$ The importance of common factor solutions was stressed in (Evans and Honkapohja 1986). For other applications see (Evans and Honkapohja 1994a) and (Evans and Honkapohja 2002b). 
with $\xi_{t} \equiv 0$, and the other representation is given by $y_{t}=a_{j} y_{t-1}+\xi_{t}+$ $\left(\beta a_{i}\right)^{-1} v_{t}$ with $\xi_{t}=a_{i} \xi_{t-1}+\left\{\left(\beta a_{j}\right)^{-1}-\left(\beta a_{i}\right)^{-1}\right\} v_{t}$.

Remark 3: In the initialized case the analog of the $\mathrm{CF}$ representation of an REE $y_{t}$ is

$$
y_{t+1}=a_{i} y_{t}+\xi_{t+1}+\left(\beta a_{j}\right)^{-1} v_{t+1}+K a_{j}^{t+1}
$$

for $t=0,1,2, \ldots$, and $K$ is arbitrary. Since $\left|a_{j}\right|<1$ as $t \rightarrow \infty$ the solution $y_{t}$ converges to a process that satisfies (8).

\section{$4.2 \quad$ T-maps}

A useful way to analyze representations of REE's is to view them as fixed points of a map. Specifically, assume agents know the functional form of the representation, but do not know the corresponding parameter values; we assume they have some perception of what the parameter values are and we call the subsequent functional form with these values imposed the perceived law of motion (PLM). Agents use the PLM to form their expectations of future values of $y_{t}$. The actual law of motion (ALM) is obtained by inserting these expectations into the reduced form model. Provided the PLM is wellspecified, the ALM will have the same functional form and the associated parameter values, which we think of as the actual parameter values, will depend on the agents' perceptions. If $\theta$ represents the agents' perceived parameter values, let $T(\theta)$ represent the actual parameter values. Then a fixed point of this $T-$ map determines an REER.

To analyze the REER's of our model, consider the case of real roots and let $\xi_{t}$ be an observable stationary exogenous process defined by

$$
\xi_{t}=\lambda \xi_{t-1}+\varepsilon_{t},
$$

where $E_{t} \varepsilon_{t+1}=0$ and $|\lambda|<1$. We assume that $\lambda$ is known. Particular values of $\lambda$ will be of interest, but we do not at this stage impose any additional restrictions on $\lambda$. In line with the preceding results we consider PLMs of the form

$$
y_{t}=a y_{t-1}+b y_{t-2}+c+d \xi_{t}+k v_{t}+l v_{t-1} .
$$

In terms of the general notation above we are thus setting

$$
\theta=(a, b, c, d, k, l)
$$


Note that this specification for the PLM includes the general form solutions (provided $\lambda=0$ ) and the common factor solutions (provided $\lambda=a_{j}$ ).

For this PLM expectations are formed according to

$$
\begin{aligned}
E_{t}^{*} y_{t+1} & =c+a E_{t}^{*} y_{t}+b y_{t-1}+d \lambda \xi_{t}+l v_{t} \\
& =c+a\left(c+a y_{t-1}+b y_{t-2}+d \xi_{t}+k v_{t}+l v_{t-1}\right)+b y_{t-1}+d \lambda \xi_{t}+l v_{t}
\end{aligned}
$$

so that

$$
E_{t}^{*} y_{t+1}=c(1+a)+\left(a^{2}+b\right) y_{t-1}+a b y_{t-2}+d(a+\lambda) \xi_{t}+(l+a k) v_{t}+a l v_{t-1} .
$$

Note that here we make the assumption, frequently made in the literature, that $y_{t}$ is not part of the time $t$ information set, but that exogenous variables $\xi_{t}$ and $v_{t}$ and lagged variables are known when forecasts are made at $t$. (Section 7 discusses the impact of including $y_{t}$ in the information set).

Substituting (11) into (1) yields the ALM

$$
\begin{aligned}
y_{t}= & \left(\beta\left(a^{2}+b\right)+\delta\right) y_{t-1}+\beta a b y_{t-2}+\beta(1+a) c+\beta d(a+\lambda) \xi_{t} \\
& +(1+\beta l+\beta a k) v_{t}+\beta a l v_{t-1},
\end{aligned}
$$

and thus the T-map is given by

$$
\begin{aligned}
a & \longrightarrow \beta\left(a^{2}+b\right)+\delta, \\
b & \longrightarrow \beta a b \\
c & \longrightarrow \beta(1+a) c, \\
d & \longrightarrow \beta(a+\lambda) d, \\
k & \longrightarrow(1+\beta l+\beta a k) \\
l & \longrightarrow \beta a l .
\end{aligned}
$$

The functional form of the PLM is sufficiently general that the fixed points of this T-map will capture both the general form representations and the common factor representations. To analyze these fixed points it is helpful to begin by considering the subsystem of $T(\theta)=\theta$ corresponding to (12) and (13). Provided that the quadratic $a=\beta a^{2}+\delta$ has distinct real roots $a_{1}$ and $a_{2}$, there are three possible solutions for $(a, b)$, given by $\left(a_{1}, 0\right),\left(a_{2}, 0\right)$ and $\left(\beta^{-1},-\beta^{-1} \delta\right)$.

There are thus two cases: (I) If $(a, b)=\left(\beta^{-1},-\beta^{-1} \delta\right)$ then it is easily seen that fixed points $\theta$ must satisfy $c=0, l=-\beta^{-1}$ and that $k$ is arbitrary. If 
$\lambda=0$ then $d$ is arbitrary and if $\lambda \neq 0$ then $d=0$. (II) If $(a, b)=\left(a_{i}, 0\right)$, for $i=1,2$, then $c=l=0$ and $k=\left(\beta a_{j}\right)^{-1}$, where $a_{j} \neq a_{i}$. If $\lambda=a_{j}$ then $d$ is arbitrary and if $\lambda \neq a_{j}$ then $d=0$.

Now let

$$
S=\left\{\left(\theta^{*}, \lambda\right) \in \mathbb{R}^{7} \mid T\left(\theta^{*}\right)=\theta^{*}\right\} .
$$

We see that $S=S_{G} \cup S_{M, 1} \cup S_{M, 2} \cup S_{C F, 1} \cup S_{C F, 2}$, where

$$
\begin{aligned}
S_{G} & =\left\{(\theta, \lambda) \in S \mid a=\beta^{-1}, b=-\beta^{-1} \delta, c=0, l=-\beta^{-1}, \lambda=0\right\}, \\
S_{M, i} & =\left\{(\theta, \lambda) \in S \mid a=a_{i}, b=c=0, d=0, k=\left(\beta a_{j}\right)^{-1}, l=0, \lambda \neq a_{j}\right\} \\
S_{C F, i} & =\left\{(\theta, \lambda) \in S \mid a=a_{i}, b=c=0, k=\left(\beta a_{j}\right)^{-1}, l=0, \lambda=a_{j}\right\} .
\end{aligned}
$$

We have the following proposition.

Proposition 5 Let $X_{t}=\left[y_{t-1}, y_{t-2}, 1, \xi_{t}, v_{t}, v_{t-1}\right]^{\prime}$, and assume that the roots $a_{1}, a_{2}$ are real.

1. If $\theta \in S_{G}$ then for any martingale difference sequence $\varepsilon_{t}, y_{t}=\theta^{\prime} X_{t}$ is a general form representation.

2. If $\theta \in S_{M, i}$ or $\theta \in S_{C F, i}$ then $y_{t}=\theta^{\prime} X_{t}$ is a common factor representation.

3. If $y_{t}$ is an REE then

(a) there exists $\theta \in S_{G}$ and martingale difference sequence $\varepsilon_{t}$ so that $y_{t}=\theta^{\prime} X_{t}$.

(b) for $i=1,2$ there exists $\theta \in S_{M, i} \cup S_{C F, i}$ and martingale difference sequence $\varepsilon_{t}$ so that $y_{t}=\theta^{\prime} X_{t}$.

Given the results from Propositions 3 and 4, the only potential subtlety in proving this Proposition is the observation that if a common factor solution conditions on the process $\xi_{t}$ then there is a solution conditioning on $d \xi_{t}$ for any $d$. To see this, notice that if $\xi_{t}$ satisfies (9), then $\xi_{t}^{\prime}=d \xi_{t}$ satisfies

$$
\xi_{t}^{\prime}=a_{j} \xi_{t-1}^{\prime}-\left(\beta a_{j}\right)^{-1} v_{t}+\varepsilon_{t}^{\prime},
$$

where $\varepsilon_{t}^{\prime}=d \varepsilon_{t}-\left(\beta a_{j}\right)^{-1}(1+d) v_{t}$. 


\section{$5 \quad$ E-stability}

Let $\theta^{*}$ be a fixed point of the T-map. We say $\theta^{*}$ (and the associated REER) is E-stable provided the differential equation

$$
\frac{d \theta}{d \tau}=T(\theta)-\theta
$$

is locally asymptotically stable at $\theta^{*}$. The E-stability Principle says that if the REER is E-stable then it is learnable by a reasonable adaptive algorithm. This principle is known to be valid for least squares and closely related statistical learning rules in a wide variety of models. For a thorough discussion see (Evans and Honkapohja 2001). ${ }^{9}$

The definition of expectational stability just given is inadequate when there is a non-trivial connected set of rest points of the differential equation (18), as is the case for our model; if the $\lambda$-section of $S$ is locally connected then no point in $S$ is locally asymptotically stable. In this case the notion of E-stability is extended as follows: we say that a set of fixed points, $Q$, is E-stable provided there is a neighborhood $U$ of $Q$ so that for any $\theta_{0} \in U$ the trajectory of $\theta$ determined by the differential system (18) converges to a point in $Q$. A necessary condition for E-stability of $Q$ is that for all points $q \in Q$ the non-zero eigenvalues of the derivative $T(\theta)-\theta$ evaluated at $q$ have negative real part. In our case this necessary condition is also sufficient because the derivative of the T-map has only one zero eigenvalue; see "Proof of Proposition 7" in the Appendix for details. Abusing notation slightly, we will say that for fixed $\lambda$, the set $S_{*}$ is E-stable provided it is E-stable as a subset of $\mathbb{R}^{6} .{ }^{10}$ Finally, we shall say a representation is E-stable provided the associated set $S_{*}$ is E-stable.

Notice that since a given REE can have different representations, we should not strictly speak of learnable REEs, but instead of whether an REER is learnable. For example we will find that, for some regions of the parameter space, common factor representations of an REE are stable under learning even when the general form representation is not. It will be terminologically

\footnotetext{
${ }^{9}$ The connection between least squares learning and E-stability is established using convergence results from the stochastic approximation literature. This technique was first applied in the macroeconomics learning literature by (Marcet and Sargent 1989).

${ }^{10}$ Since $\lambda$ is exogenous and assumed known, stability in its dimension is not relevant. If $\lambda$ were not known it could be consistently estimated by a regression of $\xi_{t}$ on $\xi_{t-1}$ and E-stability conditions would be unaffected.
} 
convenient for us to describe this by saying that the common factor solution is stable while the general form solution is not. As we will see, there are also cases in which all representations of an REE are unstable.

\subsection{Instability of General Form Solution}

We begin with a result that the general form solutions, i.e. REE written in their general form representation, are not E-stable.

Let $\phi=\left(\theta_{1}, \theta_{2}\right)$. Since the subsystem of the differential system (18) corresponding to $\phi_{1}$ is decoupled from the rest of the differential system, it follows that a necessary condition for E-stability of the general form solution is that the subsystem

$$
\frac{d \phi}{d \tau}=T_{\phi}(\phi)-\phi
$$

be locally asymptotically stable at $\phi^{*}=\left(\beta^{-1},-\delta \beta^{-1}\right)$, where $T_{\phi}$ is the obvious map. The derivative matrix is given by

$$
D\left(T_{\phi}\right)-I_{2}=\left[\begin{array}{cc}
2 \beta a-1 & \beta \\
\beta b & \beta a-1
\end{array}\right] \text {. }
$$

Recall that a fixed point is locally asymptotically stable provided the eigenvalues of the derivative have negative real part, or, equivalently, the derivative has negative trace and positive determinant. Evaluated at the fixed point $\phi_{1}^{*}$ we have $D\left(T_{\phi}\right)-I_{2}=\left[\begin{array}{cc}1 & \beta \\ -\delta & 0\end{array}\right]$, which has positive trace thus yielding the following result:

Proposition 6 The general form solution is not E-stable.

\subsection{Stability of Common Factor Solutions}

We now consider E-stability of CF solutions, i.e. REE written in their Common Factor representation and given by $S_{M, 1} \cup S_{M, 2} \cup S_{C F, 1} \cup S_{C F, 2}$. We again start by considering the subsystem (19). Evaluated at $\bar{\phi}_{i}=\left(a_{i}, 0\right)$ we obtain $D\left(T_{\phi}\right)-I_{2}=\left[\begin{array}{cc}2 \beta a_{i}-1 & \beta \\ 0 & \beta a_{i}-1\end{array}\right]$, which yields the stability condition $2 \beta a_{i}<1$. From the expressions for $a_{1}$ and $a_{2}$, it is immediate that 
this stability condition is satisfied for $a_{1}$ and fails for $a_{2}$. Thus any REER in $S_{M, 2} \cup S_{C F, 2}$ is not E-stable.

For the MSV solution $S_{M, 1}$ and the CF stationary sunspot equilibria $S_{C F, 2}$ the remaining E-stability conditions are obtained in the Appendix. We have the following result:

Proposition 7 The Common Factor solutions $S_{M, 2} \cup S_{C F, 2}$ are not E-stable. The conditions for E-stability of the Common Factor Solutions $S_{M, 1}$ and $S_{C F, 1}$ are as follows:

1. The $M S V$ solution $S_{M, 1}$ is E-stable when (i) $\beta<\frac{1}{2}$ or $\beta+\delta<1$ and (ii) $\beta\left(a_{1}+\lambda\right)<1$,

2. The CF solution set $S_{C F, 1}$ is E-stable when $\beta<\frac{1}{2}$ or $\beta+\delta<1$.

Figure 1 illustrates the region in $(\beta, \delta)$ space in which the Common Factor solution SSEs are E-stable. The region is obtained by simultaneously imposing the conditions for indeterminacy, that the roots be real and that conditions for E-stability of the $\mathrm{CF}$ solutions be satisfied. The resulting region is specified by $\beta<-\frac{1}{2}, \beta+\delta<-1$ and $4 \beta \delta<1$.

\section{FIGURE 1 ABOUT HERE}

We also make several remarks concerning the E-stability condition (ii) for the MSV solution $S_{M, 1}$. Recall that the CF solution set $S_{C F, 1}$ requires $\lambda=a_{2}$. Since $\beta\left(a_{1}+a_{2}\right)=1$, condition (ii) is not (quite) satisfied when the $\mathrm{CF}$ solution set is E-stable. However, note that the $\mathrm{CF}$ solution set $S_{C F, 1}$ includes a continuum of values for $d$ and that the CF solution for $d=0$ describes an REE that is identical to the corresponding MSV solution.

The other point to note in connection with condition (ii) for the MSV solution is that it would not be required if the PLM (10) excluded the variable $\xi_{t}$. In that case E-stability of $S_{M, 1}$ is governed by (i) and it can be seen that this condition is always satisfied throughout the regular region $|\beta+\delta|<1$, as well as in parts of the irregular and explosive regions. Condition (ii) provides an additional requirement that guarantees stability of this MSV solution even if agents condition also on $\xi_{t}$. Further discussion is given in the appendix. 


\subsection{Discussion}

Our results show that stationary sunspot equilibria are E-stable in a subset of the indeterminacy (or "irregular model") region. This is true even though the set of general form representations (7), which take an $\operatorname{ARMA}(2,1)$ form, always fail to be E-stable in this model. ${ }^{11}$ The key to obtaining E-stability in this subregion is that agents use a perceived law of motion in which they condition forecasts, not only on the predetermined variable, but also on an exogenous $\operatorname{AR}(1)$ process $\xi_{t}$ with autoregressive parameter $\lambda=a_{2}$. This exogenous process can be thought of as having a "resonant frequency," i.e. having time-series properties that are exactly right for exciting a stationary sunspot equilibrium. When the conditions $\beta<-\frac{1}{2}, \beta+\delta<-1$ and $4 \beta \delta<1$ are satisfied, conditioning learning on $\xi_{t}$ leads to an E-stable set of SSEs. These SSEs have the same time-series properties as the $\operatorname{ARMA}(2,1)$ solutions, because they are an alternative representation of these solutions.

The term "resonant frequency" was used in (Evans and Honkapohja $2002 \mathrm{~b}$ ) to describe the (well-known) condition on transition probabilities required for existence of finite state Markov SSEs in forward-looking models. Here we see that the phenomenon is in fact much more general and applies also to models with predetermined variables. An exogenous $\mathrm{AR}(1)$ process with an autocorrelation parameter equal to one of the two roots can form the basis of a sunspot equilibrium for a PLM that conditions on this exogenous variable as well as its own lag. These are the CF representations of SSEs. Furthermore, in the subset of the irregular region shown in Figure 1, conditioning on the resonant frequency sunspot variable $\xi_{t}=a_{2} \xi_{t-1}+\varepsilon_{t}$ leads to SSEs that are E-stable and are therefore (as we verify below) stable under least-squares learning.

\section{Simulations of Real-Time Learning}

Our conjecture is that for values of $\beta$ and $\delta$ in the indicated region of Figure 1, the corresponding CF solutions are stable under least squares learning. We have shown that in this area the conditions for E-stability are satisfied for the common factor representation of SSEs. The E-stability principle says that if

\footnotetext{
${ }^{11}$ In some linear models the general form solutions can be E-stable. This was found in (Evans and Honkapohja 1992), (Duffy 1994) and (Evans and Honkapohja 1994a) for models that rely on different information sets and include forecasts over multiple horizons.
} 
an REER is E-stable then it is learnable by a reasonable learning algorithm. However, the E-stability principle is only known to formally apply to REER which are isolated rest points of the differential equation (18). To support our focus on E-stability we now simulate least squares learning of the REER.

Before presenting the numerical results we briefly outline the framework under which the simulations are generated. Under least squares learning agents are assumed to have a PLM in which $y_{t}$ depends linearly on predetermined and exogenous variables $X_{t}$ with coefficients $\theta$. The values of $\theta$ are unknown to the agents, who therefore use an estimate of its value. More specifically, we assume that agents make forecasts based on the PLM (10). At the beginning of period $t$ agents have available observations of $X_{t}=\left[y_{t-1}, y_{t-2}, 1, \xi_{t}, v_{t}, v_{t-1}\right]^{\prime}$ and use the estimate $\theta_{t-1}$ of $\theta=[a, b, c, d, k, l]^{\prime}$ based on data through period $t-1$. Their forecasts are thus given by (11) with $\theta$ replaced by $\theta_{t-1}$. The value of $y_{t}$ is then determined according to (1) by these expectations and by $y_{t-1}$ and $v_{t}$. The outcome can be conveniently expressed as $y_{t}=T\left(\theta_{t-1}\right)^{\prime} X_{t}$ where $T(\theta)$ is specified by (12)-(17).

We then move to the next period $t+1$, at the beginning of which agents update their least squares estimate of $\theta$ using the time $t$ data point. The recursive least squares (RLS) learning algorithm ${ }^{12}$ for the updated coefficients of the least squares regression of $y_{t}$ on $X_{t}$ is given by

$$
\begin{aligned}
\theta_{t} & =\theta_{t-1}+\frac{1}{t} R_{t}^{-1} X_{t}\left(y_{t}-\theta_{t-1}{ }^{\prime} X_{t}\right), \\
R_{t} & =R_{t-1}+\frac{1}{t}\left(X_{t} X_{t}^{\prime}-R_{t-1}\right) .
\end{aligned}
$$

Note that $y_{t}-\theta_{t-1}{ }^{\prime} X_{t}$ is the period $t$ residual for the estimated relationship using the previous period's estimate $\theta_{t-1}$. Using $y_{t}=T\left(\theta_{t-1}\right)^{\prime} X_{t}$ the $\theta_{t}$ recursion can be written as

$$
\theta_{t}=\theta_{t-1}+\frac{1}{t} R_{t}^{-1} X_{t}\left(\left(T\left(\theta_{t-1}\right)-\theta_{t-1}\right)^{\prime} X_{t}\right) .
$$

The sequence of events is then again repeated: exogenous variables $v_{t+1}$ and $\xi_{t+1}$ are then determined, agents form expectations and $y_{t+1}$ is determined. The question of interest is whether $\theta_{t} \rightarrow \theta \in S$ as $t \rightarrow \infty$. In particular if $\theta_{t} \rightarrow \theta \in S_{G} \cup S_{C F, 1} \cup S_{C F, 2}$ then the process $y_{t}$ converges asymptotically to an SSE.

\footnotetext{
${ }^{12}$ See, for example pp. 32-3 of (Evans and Honkapohja 2001).
} 
Note that we have chosen the functional form of the PLM (10) so that the associated T-map has as fixed points all REER of interest. The generality of this form implies that for a given representation the PLM may be over-parameterized so that the associated fixed point has zeros in some entries. This over-parameterization poses no problem for E-stability; however, there may be important consequences for the corresponding real time learning algorithm. Specifically, if the over-parametrization leads to severe multicollinearity, the statistical learning algorithm may settle on a vector of parameters not corresponding to a fixed point of the T-map. Interestingly, even if this occurs, the associated sequence of endogenous variables still converges to a REE. We begin by analyzing this phenomenon in more detail and then consider the behavior of real time learning algorithms using regressors which do not yield multicollinearity problems.

\subsection{Over-Parameterization and Multicollinearity}

This issue is most easily examined by considering representations without sunspots. Specifically, we assume the following PLM:

$$
y_{t}=a y_{t-1}+b y_{t-2}+k v_{t}+l v_{t-1} \text {. }
$$

The associated T-map is given by (12), (13), (16), and (17). A fixed point of this T-map is given by $a=a_{1}, b=0, k=\left(1-\beta a_{1}\right)^{-1}$, and $l=0$, yielding a representation of the form

$$
y_{t}=a_{1} y_{t-1}+\left(1-\beta a_{1}\right)^{-1} v_{t} .
$$

Arguments already given show that for appropriate parameters, this representation to be E-stable; however, real time learning algorithms can settle on parameter values other than those yielding this representation. This is illustrated in Figure 2, which gives the results of one simulation of the model under least squares learning for this PLM with $\beta=-3$ and $\delta=1$. $^{13}$

\section{FIGURE 2 ABOUT HERE}

\footnotetext{
${ }^{13}$ In the simulations shown in Figures 2 and $3 \theta_{t}, R_{t}$ evolve according to (22) and (21), but with $X_{t}=\left[y_{t-1}, y_{t-2}, v_{t}, v_{t-1}\right]^{\prime}$ and $\theta=[a, b, k, l]^{\prime}$ and the T-map restricted as indicated. Similary in Figure $3 X_{t}=\left[y_{t-1}, y_{t-2}, v_{t}\right]^{\prime}$ and $\theta=[a, b, k]^{\prime}$.
} 
To understand this behavior, first notice that if the process $y_{t}$ solves (24) and $\eta \in \mathbb{R}$ then $y_{t}$ also solves

$$
(1-\eta L)\left(1-a_{1} L\right) y_{t}=\left(1-\beta a_{1}\right)^{-1}(1-\eta L) v_{t}
$$

This $\operatorname{ARMA}(2,1)$ process is consistent with the agents' PLM. If $y_{t}$ solves $(24)$ and agents believe in the $\operatorname{ARMA}(2,1)$ process then their regression residuals are zero. (See Lemma 8 below.) Since RLS learning relies on these residuals to update the estimator, it follows that in this case the estimator will be stuck at a point which does not correspond to a REER. Note, however, that the process $y_{t}$ is an REE.

Lemma 8 Let $\eta \in \mathbb{R}, T$ be the T-map associated with the PLM (23), and let

$$
\begin{aligned}
\theta & =\left[a_{1}+\eta,-a_{1} \eta,\left(1-\beta a_{1}\right)^{-1},-\eta\left(1-\beta a_{1}\right)^{-1}\right]^{\prime}, \\
X_{t} & =\left[y_{t-1}, y_{t-2}, v_{t}, v_{t-1}\right]^{\prime} .
\end{aligned}
$$

If $y_{t}$ satisfies (24) then $(T(\theta)-\theta)^{\prime} X_{t}=0$.

Intuitively this Lemma must hold: if $y_{t}$ satisfies (24) then $y_{t}$ satisfies (25); if agents know $y_{t}$ satisfies (25) then agents forecast $y_{t}$ correctly; if agents forecast $y_{t}$ correctly then $T(\theta)^{\prime} X_{t}=\theta^{\prime} X_{t}$. The formal proof is straightforward but tedious algebra and is omitted.

The central problem with the over-parameterization is that it leads to severe multicollinearity. Notice that if $y_{t}$ solves (24) then the regressors in the $\operatorname{ARMA}(2,1)$ representation display perfect linear correlation; $y_{t-1}$ is a linear combination of $y_{t-2}$ and $v_{t-1} \cdot{ }^{14}$ To avoid this problem, it is natural to drop one or more explanatory variables. For example, if the regressors are taken to be $y_{t-1}, y_{t-2}$, and $v_{t}$, then convergence of the RLS estimator to the MSV solution (24) does obtain, see Figure 3.

\section{FIGURE 3 ABOUT HERE}

Similarly, one could drop $y_{t-2}$ and include $v_{t-1}$ or simply choose to drop both extra regressors.

\footnotetext{
${ }^{14}$ Indeed if one extends the simulation shown in Figure 2 for a sufficiently long period, the matrix $R_{t}$ eventually becomes close enough to singularity to result in numerical breakdown of the algorithm.
} 


\subsection{Instability of General Form Solutions}

Recall that general form solutions are given by

$$
y_{t}=\beta^{-1} y_{t-1}-\delta \beta^{-1} y_{t-2}-\beta^{-1} v_{t-1}+\varepsilon_{t}
$$

where $\varepsilon_{t}$ is any martingale difference sequence and is taken to be observed. The form of the model requires $v_{t}$ to be a regressor in the agent's PLM, even though it is not present in the general form solution. (We are thus forced to specify a PLM which can sometimes lead to severe multicollinearity problems.) Specifically, we assume a PLM of the form (10) with $\lambda=0$ so that $\xi_{t}=\varepsilon_{t}$ :

$$
y_{t}=a y_{t-1}+b y_{t-2}+c+d \varepsilon_{t}+k v_{t}+l v_{t-1} \text {. }
$$

Set $X_{t}=\left[y_{t-1}, y_{t-2}, 1, \varepsilon_{t}, v_{t}, v_{t-1}\right]^{\prime}$, and $\theta=[a, b, c, d, k, l]^{\prime}$. The behavior of the algorithm (22) and (21) is analyzed via simulations. The algorithm was initialized by choosing point at random within a given neighborhood of the set $S_{G}$.

As predicted by the E-stability principle, convergence of the real time estimators to the parameters associated with the general form solution does not appear to obtain; all simulations were consistent with this REER being unstable under least squares learning. Interestingly, in cases when the norms of the estimates did not diverge to infinity, the estimators appeared to settle on seemingly arbitrary values, with the exception of the estimator for $k$ which appears to converge to $\left(1-\beta a_{1}\right)^{-1}$, and $c$ and $d$ which appear to converge to zero: see Figure 4. To explain this behavior, we must turn back to the problem of over-parameterization. As mentioned above, to nest the various representations we must over-parameterize the PLM. If convergence to the general form solution obtains then the problem of severe multicollinearity will not arise; however, the general form solution is not stable and the algorithm quickly moves the estimates away from these values. As the estimates $\theta_{t}$ adjust, so too does the endogenous variable $y_{t}$; if $y_{t}$ adjusts so that it follows the common factor representation

$$
y_{t}=a_{1} y_{t-1}+\left(1-\beta a_{1}\right)^{-1} v_{t}
$$

then, as was shown in the previous subsection, the estimators will settle on an $\operatorname{ARMA}(2,1)$ process which is consistent with this $\operatorname{AR}(1)$ representation, 
that is, the agents will learn an over-specified model. In each case in which a simulation resulted in convergence to some fixed values for the estimates, the associated $\operatorname{ARMA}(2,1)$ process was an over-parameterization of the $\operatorname{AR}(1)$ common factor solution produced above; for the simulations in Figure 4, $\eta \approx-.6623$ works.

\section{FIGURE 4 ABOUT HERE}

We conclude that although the general form REER is not stable under RLS learning, the agents may still learn an REE. Also, the learned REE will not depend on the sunspot $\varepsilon_{t}$.

\subsection{Stability of CF Solutions}

Here we analyze numerically the real-time learning implications of Proposition 7. Recall that E-stable common factor representations have the form

$$
\begin{aligned}
& y_{t}=a_{1} y_{t-1}+d \xi_{t}+\left(1-\beta a_{1}\right)^{-1} v_{t} \\
& \xi_{t}=\lambda \xi_{t-1}+\varepsilon_{t}
\end{aligned}
$$

where $\lambda$ is taken as known. ${ }^{15}$ We implement real-time learning by assuming a PLM of the form

$$
y_{t}=a y_{t-1}+c+d \xi_{t}+k v_{t}+l v_{t-1} .
$$

We have dropped the regressor $y_{t-2}$ to avoid problems with multicollinearity. Setting $\theta=[a, c, d, k, l]^{\prime}$ and $X_{t}=\left[y_{t-1}, 1, \xi_{t}, v_{t}, v_{t-1}\right]^{\prime}$ results in an updating algorithm of the form (22) and (21). We consider the sets $S_{M, 1}$ and $S_{C F, 1}$ separately.

\subsubsection{MSV-Solutions}

The E-stability Principle, together with Proposition 7, predicts that if $\beta<$ $1 / 2$, or $\beta+\delta<1$, and if $\beta\left(a_{1}+\lambda\right)<1$, then the RLS learning algorithm described about should converge locally to the MSV solution. To test this principle, we chose different parameter values satisfying these conditions and

\footnotetext{
${ }^{15}$ The assumption that $\lambda$ is known is made for simplicity. If $\lambda$ is unknown it can be estimated by agents and the stability conditions are unchanged.
} 
simulated the learning model using standard normal white noise for the martingale difference sequence $\varepsilon_{t}$. Initial conditions were chosen randomly within a small neighborhood of the MSV parameter values.

Analytic results implying convergence with probability one typically require amending the algorithm with a projection facility. Alternatively, one can adjust the gain of the algorithm to obtain convergence with probability approaching one. For the simulations produced here we scale the gain of the RLS algorithm by $1 / 25$, thus increasing the probability of convergence.

We found that with positive probability (that is, for a positive proportion of our simulations), convergence to the MSV solution appeared to obtain.

\section{FIGURE 5 ABOUT HERE}

Figure 5 shows the results of a simulation corresponding to the parameter values $\beta=-3, \lambda=.5$, and $\delta=1$ (so that $\beta\left(a_{1}+\lambda\right)=-2.8$ approximately). Graphs obtained using other values of $\beta, \lambda$, and $\delta$ satisfying the E-stability conditions were qualitatively similar.

\subsubsection{Common Factor Solutions}

We used simulations analogous to those mentioned above to analyze the stability of common factor sunspots for the case $\lambda=a_{2}$. Initial conditions were chosen at random near the set $S_{C F, 1}$; the initial value of $\theta_{3}$ was chosen at random near one. Proposition 7 predicts that if $\beta<1 / 2$ or $\beta+\delta<1$ then convergence to some point in $S_{C F, 1}$ will obtain. Our simulations suggest that with positive probability, this is indeed the case.

\section{FIGURE 6 ABOUT HERE}

Figure 6 shows the results of a simulation corresponding to the parameter values $\beta=-3$, and $\delta=1$. Notice, in particular, that $\theta_{3}=d$ appears to converge to some number not equal to zero, suggesting that agents in this economy will indeed learn that there is a dependence on a sunspot variable.

\section{Alternative Information Assumption}

We have assumed that, when forming expectations $E_{t}^{*} y_{t+1}$ using the PLM (10), agents have available observations of current exogenous variables $v_{t}$ 
and $\xi_{t}$ and the lagged endogenous variable $y_{t-1}$. An alternative assumption that could be made is that expectations $E_{t}^{*} y_{t+1}$ can condition also on current $y_{t}$. In this case $y_{t}$ and $E_{t}^{*} y_{t+1}$ are simultaneously determined. Although the assumptions are equivalent under rational expectations they lead to different out of equilibrium behavior and therefore can lead to different stability conditions under learning. In this Section we summarize how the stability conditions for learning SSEs are affected by including $y_{t}$ in the information set.

Expectations are now given by

$$
E_{t}^{*} y_{t+1}=a y_{t}+b y_{t-1}+c+d \lambda \xi_{t}+l v_{t}
$$

Inserting into (1) and solving for $y_{t}$ gives the ALM

$$
y_{t}=\frac{\beta b+\delta}{1-\beta a} y_{t-1}+\frac{\beta c}{1-\beta a}+\frac{\beta d \lambda}{1-\beta a} \xi_{t}+\frac{1+\beta l}{1-\beta a} v_{t}
$$

which implicitly defines the T-map. Note that the map is well defined only for $a \neq \beta^{-1}$. When $a=\beta^{-1}$ the temporary equilibrium does not exist, i.e. there is no solution for $y_{t}$ consistent with this expectation formation rule. This difficulty, which did not occur under are previous information assumption, arises here because of the simultaneity of $y_{t}$ and $E_{t}^{*} y_{t+1}{ }^{16}$ This in particular applies to the general form solutions, in which $a=\beta^{-1}$ and $b=-\beta^{-1} \delta$, but it can anyway be seen from the $b$ component of the T-map $T_{b} \equiv 0$ that the general form solutions cannot be E-stable.

We focus on the E-stability conditions for the CF solutions in the indeterminate case. The key stability conditions at the CF solutions $a=a_{i}$, for $i=1,2$, and $b=0$ are that

$$
D T_{a}=\frac{\beta \delta}{\left(1-\beta a_{i}\right)^{2}}<1 \text { and } D T_{c}=\frac{\beta}{1-\beta a_{i}}<1
$$

In the appendix we show that the E-stability conditions for the set $S_{C F, 1}$ are unchanged: this solution set is E-stable if and only if $\beta<\frac{1}{2}$ or $\beta+\delta<1$ and thus is E-stable as before in the shaded region marked in Figure 1. However, a new case arises with the set $S_{C F, 2}$. Under the earlier information assumptions this set was never E-stable, but under the current assumptions

\footnotetext{
${ }^{16}$ The possibility of nonexistence of temporary equilibrium is well known, see e.g. (Grandmont and Laroque 1990)
} 
it is E-stable in the region bounded by $\delta<0$ and $\beta+\delta>1$. We continue to find that neither $\mathrm{CF}$ solution set is E-stable for the part of the irregular region in which $\beta, \delta>0$.

These results confirm (and somewhat strengthen) our earlier results: for a strict subset of the parameter space there exist SSEs that are locally stable under learning provided agents use a PLM that nests the common factor representation of these SSEs.

\section{Investment under Uncertainty}

We develop an example based on the (Lucas and Prescott 1971) model of investment under uncertainty, extended in (Sargent 1987), Ch. XIV, to allow for market distortions due to taxes and externalities. For this model the stability under learning of the Minimal State Variable solutions was examined in (Evans and Honkapohja 2001), Section 8.6.2.

Consider a competitive industry with $N$ identical firms. Output $x_{t}$ of the representative firm at $t$ is given by

$$
x_{t}=x_{0}+f_{0} k_{t}+f_{1} K_{t}+f_{2} K_{t-1},
$$

where $k_{t}$ is the capital stock of the individual firm and $K_{t}=N k_{t}$ denotes the aggregate capital stock. The presence of the two terms in $K_{t}$ reflect contemporaneous and lagged external effects. These may be positive or negative, so we do not restrict the signs of $f_{1}$ or $f_{2}$, but $f_{0}>0$ and $x_{0}>0$. Taxes are levied on firms on capital in place. The rate itself is assumed to depend on current and lagged aggregate capital stock, so that $\tau_{t}=g_{0}+g_{1} K_{t}+g_{2} K_{t-1}$. Total output is given by $X_{t}=N x_{t}$, and demand for the market is

$$
p_{t}=D-A X_{t}+u_{t}
$$

where $u_{t}$ is white noise. We require $p_{t} \geq 0$.

The firm chooses $k_{t}$ to maximize

$$
E_{0} \sum_{t=0}^{\infty} B^{t}\left\{p_{t}\left(x_{0}+f_{0} k_{t}+f_{1} K_{t}+f_{2} K_{t-1}\right)-w k_{t}-\tau_{t} k_{t}-\frac{C}{2}\left(k_{t}-k_{t-1}\right)^{2}\right\},
$$

where $k_{-1}$ is given and $w$, the rental on capital goods, is for convenience assumed to be constant. $C>0$ reflects adjustment costs for changing $k_{t}$. 
The Euler equation for this problem can be written

$$
p_{t} f_{0}-\left(w+\tau_{t}\right)+B C E_{t}^{*} k_{t+1}-C(1+B) k_{t}+C k_{t-1}=0
$$

for $t \geq 0$. For an optimum solution for the firm we also require that $k_{t} \geq$ $0, x_{t} \geq 0$, and that the transversality condition is met.

In order to define the temporary equilibrium and study learning, we need to be careful about the information structure. We assume that firms use observations of lagged capital stock, the current intrinsic exogenous shocks $u_{t}$ and the current extrinsic exogenous variable $s_{t}$ to make forecasts $E_{t}^{*} k_{t+1}$. Given these forecasts, firms choose their demands for capital $k_{t}$, conditional on $p_{t}$ and $\tau_{t}$, to satisfy (26). The temporary equilibrium is then given by the market clearing values of $p_{t}, \tau_{t}$ and $k_{t}$. Using the identical agent assumption, and combining equations, we obtain the reduced form

$$
k_{t}=\alpha+\beta E_{t}^{*} k_{t+1}+\delta k_{t-1}+\gamma u_{t},
$$

where $\beta=B C \Omega^{-1}, \delta=-\left(f_{0} A f_{2} N^{2}+g_{2} N-C\right) \Omega^{-1}, \Omega=f_{0} A N\left(f_{0}+f_{1} N\right)+$ $g_{1} N+C(1+B)$ and $\gamma=\Omega^{-1}$. With no externalities or taxes there is a unique stationary REE. However, in general the parameters $\beta$ and $\delta$ are unrestricted. In particular, for some parameter regions the associated quadratic has both real roots inside the unit circle, and there are multiple stationary solutions, including solutions depending on the extraneous sunspot variable $s_{t}$.

Several further remarks on this set-up are in order. In line with the treatment in the main body of this paper we are assuming that, although exogenous variables at $t$ are observable, data on aggregate capital $K_{t}$ are not available at the time forecasts $E_{t}^{*} k_{t+1}$ are made. ${ }^{17}$ Second, firms are choosing $k_{t}$ on the basis of their Euler equation (26), and to do so they require forecasts of their own decision the following period, given available information and their current decision rule. This is one natural way to formulate the temporary equilibrium with learning and it has the advantage of keeping close to the existing literature on adaptive learning. Third, the assumption of identical agents considerably simplifies the problem, since aggregate and firm capital do not then separately enter the reduced form. Adding, for example, firm specific productivity shocks would lead to a reduced form with separate dependencies on lagged aggregate and firm capital. It should be

\footnotetext{
${ }^{17}$ If expectations $E_{t}^{*} k_{t+1}$ can be made conditional on $K_{t}$ then the results of Section 7 would be applicable. Other information assumptions could also be envisaged and could be studied using analogous techniques.
} 
clear, however, that the methodology of this paper could also be extended to such frameworks.

Forecasts $E_{t}^{*} k_{t+1}$ are assumed to be based on a PLM of the form

$$
k_{t}=a k_{t-1}+b k_{t-2}+c+d \xi_{t}+k u_{t}+l u_{t-1},
$$

where under real time learning the coefficients are updated by recursive least squares. ${ }^{18}$ Here $\xi_{t}$ is an exogenous stationary $\operatorname{AR}(1) \xi_{t}=\lambda \xi_{t-1}+\varepsilon_{t}$. This PLM nests all of the RE solutions, as we have shown, for appropriate choices of $\lambda$. We are now entirely in the framework that we have studied in this paper.

This model is easy to study numerically. As noted above, when externality or tax distortions are present the indeterminacy case is possible. It is easily verified that various ranges of structural parameter values yield reduced form parameters in the subregion in which the Common Factor representation of SSEs is stable under learning. For example, normalizing with $N=1$, the parameter values $A=1, B=0.95, C=0.46, f_{0}=1 . f_{1}=-1, f_{2}=0.3$, $g_{1}=-1.043, g_{2}=0.306$, leads approximately to $\beta=-3$ and $\delta=1$.

The possibility that SSEs in this model can be stable under learning has not previously been noticed. Our results suggest that research into the empirical plausibility of these cases would be of considerable interest.

\section{Conclusion}

We have studied in detail the stability under learning of stationary sunspot equilibria (SSEs) in the linear stochastic univariate expectations model with a predetermined variable. In the irregular case in which SSEs exist there are alternative representations of the solutions, and we have shown that the representation is crucial for the stability of SSEs under learning. An SSE can be represented (in "general form") as an $\operatorname{ARMA}(2,1)$ process depending on an exogenous martingale difference sequence. When agents use such representations to estimate the law of motion to generate their forecasts, none of the SSEs are stable under learning. However, SSEs have an alternative "common factor" representation, and when agents use this perceived law of

\footnotetext{
${ }^{18}$ Because of our representative agent assumption, lagged aggregate capital $K_{t-1}$ and/or $K_{t-2}$ should not be additionally included. These variables do not incorporate any additional information and if included would lead to extreme multicollinearity in estimates of the PLM parameters.
} 
motion, the SSEs are locally stable under learning in a strict subset of the parameter space. We have illustrated and extended our theoretical results using numerical simulations and provided an economic example based on investment under uncertainty in a competitive market with distortions. In the Introduction we emphasized the importance in applied macroeconomics of multivariate expectations models with predetermined variables. Given their potential importance to business cycle theory, the extension of the findings of this paper to multivariate models is a high priority for our current research. 


\section{Appendix}

\subsection{Stationarity and Boundedness in Mean}

We here carefully state and prove the results described in Section 2. We begin with notation and definitions.

A stochastic process is a countable collection of random variables $y_{t}$ defined on a given probability space $(\Omega, \mathcal{F}, \mu)$. If the process is defined for $t \geq t_{0}$ we say that the process is initialized, otherwise we say the process is doubly infinite. Given the process $y_{t}$, define $\mathcal{F}_{t} \subset \mathcal{F}$ to be the smallest $\sigma$-field so that $y_{s}$ is measurable for all $s \leq t$. Note that $\mathcal{F}_{t} \subset \mathcal{F}_{t+1}$. We now recall the definition of conditional expectation: $E_{t} y_{t+s}: \Omega \rightarrow \mathbb{R}$ is the almost everywhere unique $\mathcal{F}_{t}$-measurable function so that for all $\Lambda \in \mathcal{F}_{t}$ we have

$$
\int_{\Lambda} y_{t+s} d \mu=\int_{\Lambda} E_{t} y_{t+s} d \mu
$$

We now make the following definitions: We say that the process $y_{t}$ is Uniformly Bounded in Expectation (UB) provided

$$
\sup _{t} E\left|y_{t}\right|<\infty
$$

We say the process is Conditionally Uniformly Bounded in Expectation (CUB) provided that for all $t$

$$
\sup _{s} E_{t}\left|y_{t+s}\right|<\infty \quad \text { a.e. }
$$

We have the following lemma.

Lemma 9 If the process is uniformly bounded in expectation then it is conditionally uniformly bounded in expectation.

Proof. We prove the contra-positive. Assume the process is not CUB. WLOG, $y_{n} \geq 0$. Then for some $n$ we have that at least one of the following two statements must hold: $\exists B \in \mathcal{F}_{n}$ of positive measure such that

1. $\lim _{s \rightarrow \infty} E_{n} y_{n+\tau(s)}=\infty$

2. $\lim _{s \rightarrow \infty} E_{n} y_{n-\tau(s)}=\infty$ 
on $B$ for some subsequence as defined by the monotonic increasing function $\tau: \mathbb{N} \rightarrow \mathbb{N}$. In the first case, we proceed as follows: that (1) holds implies that for any positive real numbers $M$ and $\delta$, there exists $\mathcal{F}_{n}$-measurable $\hat{B} \subset B$ and positive integer $N$ so that $\mu(\hat{B})>\mu(B)-\delta$ and $E_{n} y_{n+\tau(s)} \geq M$ on $\hat{B}$ for all $s \geq N$. Now notice $s \geq N$ implies

$$
E\left(y_{n+\tau(s)}\right) \geq \int_{\hat{B}} y_{n+\tau(s)} d \mu=\int_{\hat{B}} E_{n} y_{n+\tau(s)} d \mu \geq M(\mu(B)-\delta)
$$

where the middle equality follows from the definition of the conditional expectations operator. Since $\delta$ was arbitrarily small and $M$ was arbitrarily large, we have that the unconditional expectation is unbounded. To address the second case simply notice the same argument holds. Of course, in this case, for $s \leq 0, E_{n} y_{n+s}=y_{n+s}$.

The converse to the above result is false. As a simple example, let $x$ be any positive random variable with infinite expectation: for example, let $z$ be uniformly distributed on $(0,1)$ and let $x=1 /(1-z)$. Now let $y_{t}=x$. Then $E_{t} y_{t+s}=x$ so that the process is CUB, but $E y_{t}=\infty$ so that the process is not UB. Notice this argument applies to both initialized and doubly infinite processes.

We may now extend the comparison to stationary and asymptotically stationary processes. Let $\left\{y_{t}\right\}$ be a stochastic process and $\gamma_{j t}$ the $j^{\text {th }}$-autocovariance of $y_{t}$. If $y_{t}$ is doubly infinite then it is covariance stationary (CS) provided E $E y_{t}$ and $\gamma_{j t}$ exist and are independent of time for all $t$ and $j$. If $y_{t}$ is an initialized process then it is asymptotically covariance stationary (ACS) provided Eyt and $\gamma_{j t}$ exist and the following hold:

1. $\lim _{s \rightarrow \infty} E y_{s}<\infty$

2. $\lim _{s \rightarrow \infty} \gamma_{j s}<\infty \forall j \in \mathbb{N}$.

The second condition in particular requires that $\lim _{s \rightarrow \infty} \gamma_{0 s}<\infty$. It is well known that $E|x|^{p}<\infty$ implies $E|x|^{p-1}<\infty$ for any random variable $x$ and for all $p>1$. Hence $\lim _{s \rightarrow \infty} \gamma_{0 s}<\infty$ implies $\lim _{s \rightarrow \infty} E\left|y_{s}\right|<\infty$. Thus CS or ACS implies UB. To see that UB does not imply CS or ACS, consider the following deterministic process

$$
y_{t}= \begin{cases}1 & \text { if } \mathrm{t} \text { is even } \\ 0 & \text { if } \mathrm{t} \text { is odd }\end{cases}
$$

This process is clearly UB and neither stationary nor asymptotically stationary. We summarize the above results in the following proposition. 


\section{Proposition 10 1. If $y_{t}$ is a doubly infinite process then}

(a) CS implies UB implies CUB.

(b) CUB does not imply UB does not imply $C S$.

2. If $y_{t}$ is an initialized process then

(a) ACS implies UB implies CUB.

(b) CUB does not imply UB does not imply ACS.

\subsection{Proof of Proposition 1}

Any solution of (1) can be written in the form (6). Considering first the regular case, we can factor $A$ as $A=Q\left(\begin{array}{cc}a_{1} & 0 \\ 0 & a_{2}\end{array}\right) Q^{-1}$, where the columns of $Q$ are the eigenvectors of $a_{1}$ and $a_{2}$ respectively. It can be verified that $Q=\left(\begin{array}{cc}a_{1} & a_{2} \\ 1 & 1\end{array}\right)$ and $Q^{-1}=\left(a_{1}-a_{2}\right)^{-1}\left(\begin{array}{cc}1 & -a_{2} \\ -1 & a_{1}\end{array}\right)$. Letting $Q^{-1}=$ $\left(\begin{array}{ll}q^{11} & q^{12} \\ q^{21} & q^{22}\end{array}\right)$ and defining $\left(\begin{array}{c}p_{t+1} \\ r_{t+1}\end{array}\right)=Q^{-1}\left(\begin{array}{c}y_{t+1} \\ y_{t}\end{array}\right),(6)$ can be rewritten as

$$
\left(\begin{array}{c}
p_{t+1} \\
r_{t+1}
\end{array}\right)=\left(\begin{array}{cc}
a_{1} & 0 \\
0 & a_{2}
\end{array}\right)\left(\begin{array}{c}
p_{t} \\
r_{t}
\end{array}\right)+\left(\begin{array}{l}
-q^{11} \beta^{-1} \\
-q^{21} \beta^{-1}
\end{array}\right) v_{t}+\left(\begin{array}{c}
q^{11} \\
q^{21}
\end{array}\right) \varepsilon_{t+1} .
$$

It follows that

$$
\begin{aligned}
& E_{t} p_{t+1}=a_{1} p_{t}-q^{11} \beta^{-1} v_{t} \text { and } E_{t} p_{t+s+1}=a_{1} E_{t} p_{t+s} \text { for } s=1,2,3, \ldots \\
& E_{t} r_{t+1}=a_{2} r_{t}-q^{21} \beta^{-1} v_{t} \text { and } E_{t} r_{t+s+1}=a_{2} E_{t} r_{t+s} \text { for } s=1,2,3, \ldots
\end{aligned}
$$

Since $\left|a_{2}\right|>1$ and $E_{t} r_{t+s}=a_{2}^{s-1} E_{t} r_{t+1}$ we have $\lim _{s \rightarrow \infty}\left|E_{t} r_{t+s}\right|=\infty$ unless $r_{t}=a_{2}^{-1} q^{21} \beta^{-1} v_{t}$. It follows that a nonexplosive solution must satisfy $y_{t}=$ $-\left(q^{22} / q^{21}\right) y_{t-1}+a_{2}^{-1} \beta^{-1} v_{t}$. Since $-\left(q^{22} / q^{21}\right)=a_{1}$ we have $y_{t}=a_{1} y_{t-1}+$ $\left(\beta a_{2}\right)^{-1} v_{t}$. There is a unique such solution that meets the initial condition and a unique such stationary solution. Therefore (ii) follows.

In the case $\left|a_{1}\right|,\left|a_{2}\right|>1$, suppose first that the roots are real. Then we still have $\left|a_{2}\right|>1$ so that imposing the associated nonexplosiveness condition implies $y_{t}=a_{1} y_{t-1}+\left(1-\beta a_{1}\right)^{-1} v_{t}$. However $\left|a_{1}\right|>1$ implies that this solution is also explosive, and hence there are no nonexplosive solutions, almost surely. 
Suppose instead that the roots are nonreal and given by $r(\cos \omega \pm i \sin \omega)$ with associated eigenvectors $m \pm i n$. We can factor $A$ as $A=W r R_{\omega} W^{-1}$, where

$$
\begin{aligned}
& R_{\omega}=\left(\begin{array}{cc}
\cos \omega & -\sin \omega \\
\sin \omega & \cos \omega
\end{array}\right) \text { and } W=\left(\begin{array}{ll}
n & m
\end{array}\right) \text {. Write } \\
& W^{-1}\left(\begin{array}{c}
y_{t+1} \\
y_{t}
\end{array}\right)=r R_{\omega} W^{-1}\left(\begin{array}{c}
y_{t} \\
y_{t-1}
\end{array}\right)+\left(\begin{array}{c}
-w^{11} \beta^{-1} \\
-w^{21} \beta^{-1}
\end{array}\right) v_{t}+\left(\begin{array}{c}
w^{11} \\
w^{21}
\end{array}\right) \varepsilon_{t+1},
\end{aligned}
$$

where $w^{i j}$ are the components of $W^{-1}$. Setting $\left(\begin{array}{c}p_{t+1} \\ s_{t+1}\end{array}\right)=W^{-1}\left(\begin{array}{c}y_{t+1} \\ y_{t}\end{array}\right)$ we have

$$
\begin{aligned}
& E_{t}\left(\begin{array}{c}
p_{t+1} \\
s_{t+1}
\end{array}\right)=r R_{\omega}\left(\begin{array}{c}
p_{t} \\
s_{t}
\end{array}\right)+\left(\begin{array}{c}
-w^{11} \beta^{-1} \\
-w^{21} \beta^{-1}
\end{array}\right) v_{t}, \\
& E_{t}\left(\begin{array}{c}
p_{t+n} \\
s_{t+n}
\end{array}\right)=r^{n-1} R_{\omega}^{n-1} E_{t}\left(\begin{array}{c}
p_{t+1} \\
s_{t+1}
\end{array}\right) .
\end{aligned}
$$

Since by assumption $r>1$, a solution is explosive unless $E_{t}\left(\begin{array}{c}p_{t+1} \\ s_{t+1}\end{array}\right)=0$, i.e.

$$
\left(\begin{array}{c}
y_{t} \\
y_{t-1}
\end{array}\right)=W r^{-1} R_{\omega}^{-1}\left(\begin{array}{c}
w^{11} \beta^{-1} \\
w^{21} \beta^{-1}
\end{array}\right) v_{t} .
$$

This implies that $y_{t}=k v_{t}$ for some $k$. But then $E_{t} y_{t+1}=0$ and $y_{t}=\delta y_{t-1}+v_{t}$. Since $\delta \neq 0$ we have arrived at a contradiction, almost surely, and (i) is established.

In the irregular case $\left|a_{1}\right|,\left|a_{2}\right|<1$, all solutions of the form (5) are nonexplosive. For $\varepsilon_{t+1}$ a stationary stochastic process the solution

$$
y_{t+1}=\left(1-\beta^{-1} L-\beta^{-1} \delta L^{2}\right)^{-1}\left(-\beta^{-1} v_{t}+\varepsilon_{t+1}\right)
$$

is stationary. If an initial condition $y_{0}=\breve{y}_{0}$ is given then all solutions of the form (5) for $t=1,2,3, \ldots$, are nonexplosive, where $y_{1}$ is arbitrary. Letting $x_{t+1}=\left(y_{t+1}, y_{t}\right)^{\prime}$ we can rewrite (5) as $x_{t+1}=A x_{t}-\beta^{-1} v_{t}+\varepsilon_{t+1}$ and hence $x_{t+1}=A^{t} x_{1}+\sum_{j=0}^{t-1} A^{j}\left(-\beta^{-1} v_{t-j}+\varepsilon_{t+1-j}\right)$, where $x_{1}=\left(y_{1}, \breve{y}_{0}\right)^{\prime}$. Consider another solution generated by the same martingale difference sequence $\varepsilon_{t+1}$ but with initial condition $x_{1}^{\prime}=\left(y_{1}^{\prime}, \breve{y}_{0}\right)^{\prime}$ with $y_{1}^{\prime} \neq y_{1}$. Then $\left|x_{t+1}^{\prime}-x_{t+1}\right|=\left|A^{t}\left(x_{1}^{\prime}-x_{1}\right)\right| \rightarrow 0$. Hence every solution is indeterminate and (iii) is established. 


\subsection{Proof of Corollary 2}

Determining the subset of the $(\beta, \delta)$ plane which corresponds to indeterminacy is simplified by considering separate cases.

Case 1: Real Eigenvalues $(\beta \delta<1 / 4)$. In this case, the eigenvalue of largest size is $a_{2}$. Subcases are required.

Subcase 1: $\beta>0$. Then

$$
\begin{gathered}
\left|a_{2}\right|<1 \quad \Leftrightarrow \\
\sqrt{1-4 \beta \delta}<2 \beta-1 \quad \Leftrightarrow \\
\beta>\frac{1}{2} \quad \text { and } \quad \delta>1-\beta .
\end{gathered}
$$

Subcase $2: \beta<0$. Then

$$
\begin{gathered}
\left|a_{2}\right|<1 \quad \Leftrightarrow \\
\sqrt{1-4 \beta \delta}<-2 \beta-1 \quad \Leftrightarrow \\
\beta<-\frac{1}{2} \quad \text { and } \quad \delta<-1-\beta .
\end{gathered}
$$

Case 2: Complex Eigenvalues $(\beta \delta \geq 1 / 4)$. In this case the eigenvalues have the same modulus. We have

$$
\begin{aligned}
& \left|a_{2}\right|<1 \Leftrightarrow \\
& \left(\frac{1}{2 \beta}\right)^{2}+\left(\frac{\sqrt{4 \beta \delta-1}}{2 \beta}\right)^{2}<1 \Leftrightarrow \\
& \{\beta>0 \text { and } \delta<\beta\} \quad \text { or } \quad\{\beta<0 \text { and } \delta>\beta\}
\end{aligned}
$$

\subsection{E-stability Conditions for CF Solutions}

\subsubsection{Proof of Proposition 7}

The main text shows that in the differential equation for $(a, b)$ the fixed point $\left(a_{2}, 0\right)$ is locally unstable and hence that $S_{M, 2}$ and $S_{C F, 2}$ are E-unstable. The main text also shows that $\left(a_{1}, 0\right)$ is locally stable. We now obtain the additional E-stability conditions for $S_{M, 1}$ and $S_{C F, 1}$. The differential equations in $(a, b, c)$ have a recursive structure since $a$ and $b$ are independent of $c$. The equation in $c$ thus gives the additional stability condition $\beta\left(1+a_{1}\right)<1$, which is equivalent to $2 \beta-1<\sqrt{1-4 \beta \delta}$. Clearly this is satisfied for $\beta<\frac{1}{2}$ 
and for $\beta \geq \frac{1}{2}$ it is satisfied if $(2 \beta-1)^{2}<1-4 \beta \delta$, which is equivalent to $\beta+\delta<1$. The differential equations in $(a, b, k, l)$ also have a recursive structure and since $\beta a_{1}<1$ it is easily verified that $(k, l) \rightarrow\left(\left(1-\beta a_{1}\right)^{-1}, 0\right)$. This leaves the additional conditions required for convergence of the component $d$. Again the system $(a, b, d)$ is recursive with $a, b$ independent of $d$. For the MSV solution $S_{M, 1}$, because $a \rightarrow a_{1}$, the additional E-stability condition is given by $\beta\left(a_{1}+\lambda\right)<1$. For the CF solution $S_{C F, 1}$ we have $\lambda=a_{2}$ and $\beta\left(a_{1}+\lambda\right)=1$. For this case the differential equation for $d$ is given by $d / d \tau(d(\tau))=(\beta(a+\lambda)-1) d(\tau)$. Using an argument analogous to (Evans and Honkapohja 1992), p. 6, it can be shown that $a(\tau) \rightarrow a_{1}$ implies that $d(\tau)$ converges to a finite value, and no additional stability conditions are required.

\subsubsection{Discussion of E-stability conditions for $S_{M, 1}$}

We discuss briefly the condition $\beta\left(a_{1}+\lambda\right)<1$ required for E-stability of the MSV solution $S_{M, 1}$. Because $\beta\left(1+a_{1}\right)<1$ is also required for E-stability, and because $\lambda<1$, the condition $\beta\left(a_{1}+\lambda\right)<1$ is satisfied whenever $\beta>0$. In the case $\beta<0$ we need $2 \beta \lambda-1<\sqrt{1-4 \beta \delta}$. It can be shown that this always holds if $\beta>-\frac{1}{2}$ or if $\beta+\delta>-1$. In the remaining region, namely $\beta<-\frac{1}{2}$ and $\beta+\delta<-1$, E-stability requires that $\lambda>a_{2}$.

\subsubsection{E-stability conditions under alternative information assump- tion}

For the T-map given in Section 7, E-stability of a CF solution set requires that $D T_{a}=\frac{\beta \delta}{\left(1-\beta a_{i}\right)^{2}}<1$ and $D T_{c}=\frac{\beta}{1-\beta a_{i}}<1$. It can be verified that $D T_{a}\left(a_{1}\right)<1$ is always satisfied and that $D T_{a}\left(a_{2}\right)<1$ if and only if $\beta \delta<0$. For $D T_{c}$ it can be shown that $D T_{c}\left(a_{1}\right)<1$ if and only if $\beta \leq \frac{1}{2}$ or $\beta+\delta<1$ and that $D T_{c}\left(a_{2}\right)<1$ if and only if either $\beta>\frac{1}{2}$ or $\left(0<\beta<\frac{1}{2}\right.$ and $\left.\beta+\delta<1\right)$ or $(\beta<0$ and $\beta+\delta>1)$. Within the irregular regions these conditions lead to the subregions of E-stability given in the text. Note that the differential equation for $d$ is given by $d / d \tau(d(\tau))=\left((1-\beta a)^{-1} \beta \lambda-1\right) d(\tau)$. Again the coefficient $(1-\beta a)^{-1} \beta \lambda-1=0$ at the CF solution $a=a_{i}, \lambda=a_{j}$, corresponding to the continuum in $d$. Since the system $(a, b, d)$ is recursive with $a, b$ independent of $d$ it again follows that $a(\tau) \rightarrow a_{1}$ implies that $d(\tau)$ converges to a finite value. 


\section{References}

Azariadis, C. (1981): "Self-Fulfilling Prophecies," Journal of Economic Theory, 25, 380-396.

Barnett, W., J. Geweke, and K. Shell (eds.) (1989): Economic Complexity: Chaos, Sunspots, Bubbles, and Nonlinearity. Cambridge University Press, Cambridge.

Benhabib, J., and R. A. FArmer (1994): "Indeterminacy and Increasing Returns," Journal of Economic Theory, 63, 19-41.

Blanchard, O., and C. Kahn (1980): "The Solution of Linear Difference Models under Rational Expectations," Econometrica, 48, 1305-1311.

Broze, L., C. Gourieroux, and A. Szafarz (1990): Reduced Forms of Rational Expectations Models. Harwood Academic Publishers, Chur, Switzerland.

Cass, D., And K. Shell (1983): "Do Sunspots Matter?," Journal of Political Economy, 91, 193-227.

Champsaur, P., and Others (eds.) (1990): Essays in Honor of Edmond Malinvaud, Volume 1. Microeconomics. MIT Press, Cambridge, Mass.

Chiappori, P., and R. Guesnerie (1989): "On Stationary Sunspot Equilibria of Order K," in (Barnett, Geweke, and Shell 1989).

Duffy, J. (1994): "On Learning and the Nonuniqueness of Equilibrium in an Overlapping Generations Model with Fiat Money," Journal of Economic Theory, 64, 541-553.

Evans, G. W., and S. Honkapohja (1986): "A Complete Characterization of ARMA Solutions to Linear Rational Expectations Models," Review of Economic Studies, 53, 227-239.

\footnotetext{
International Economic Review, 33, 1-14.
}

(1994a): "Learning, Convergence, and Stability with Multiple Rational Expectations Equilibria," European Economic Review, 38, 1071-1098. 
(1994b): "On the Local Stability of Sunspot Equilibria under Adaptive Learning Rules," Journal of Economic Theory, 64, 142-161.

- (2001): Learning and Expectations in Macroeconomics. Princeton University Press, Princeton, New Jersey.

(2002a): "Existence of Adaptively Stable Sunspot Equilibria near an Indeterminate Steady State," Journal of Economic Theory, forthcoming.

— (2002b): "Expectational Stability of Stationary Sunspot Equilibria in a Forward-looking Linear Model," mimeo.

Farmer, R. E. (1999): The Economics of Self-Fulfilling Prophecies, Second edition. MIT Press, Cambridge, Mass.

Farmer, R. E., And J.-T. Guo (1994): "Real Business Cycles and the Animal Spirits Hypothesis," Journal of Economic Theory, 63, 42-72.

Gourieroux, C., J. Laffont, and A. Monfort (1982): "Rational Expectations in Dynamic Linear Models: Analysis of the Solutions," Econometrica, 50, 409-425.

Grandmont, J.-M., and G. Laroque (1990): "Stability, Expectations, and Predetermined Variables," in (Champsaur and Others 1990), chap. 3, pp. 71-92.

Guesnerie, R. (1986): "Stationary Sunspot Equilibria in an N-commodity World," Journal of Economic Theory, 40, 103-128.

Guesnerie, R., And M. Woodford (1992): "Endogenous Fluctuations," in (Laffont 1992), chap. 6, pp. 289-412.

Keynes, J. M. (1936): The General Theory of Employment, Interest and Money. Macmillan, London.

Laffont, J.-J. (ed.) (1992): Advances in Economic Theory: Sixth World Congress. Volume 2. Cambridge University Press, Cambridge, UK.

Lucas, JR., R. E., And E. C. Prescott (1971): "Investment under Uncertainty," Econometrica, 39, 659-681. 
Marcet, A., and T. J. Sargent (1989): "Convergence of Least-Squares Learning Mechanisms in Self-Referential Linear Stochastic Models," Journal of Economic Theory, 48, 337-368.

McCallum, B. (1983): "On Nonuniqueness in Linear Rational Expectations Models: An Attempt at Perspective," The Journal of Monetary Economics, 11, 134-168.

Sargent, T. J. (1987): Macroeconomic Theory, Second edition. Academic Press, New York.

SHELL, K. (1977): "Monnaie et Allocation Intertemporelle," Working paper, CNRS Seminaire de E.Malinvaud, Paris.

Woodford, M. (1990): "Learning to Believe in Sunspots," Econometrica, $58,277-307$. 


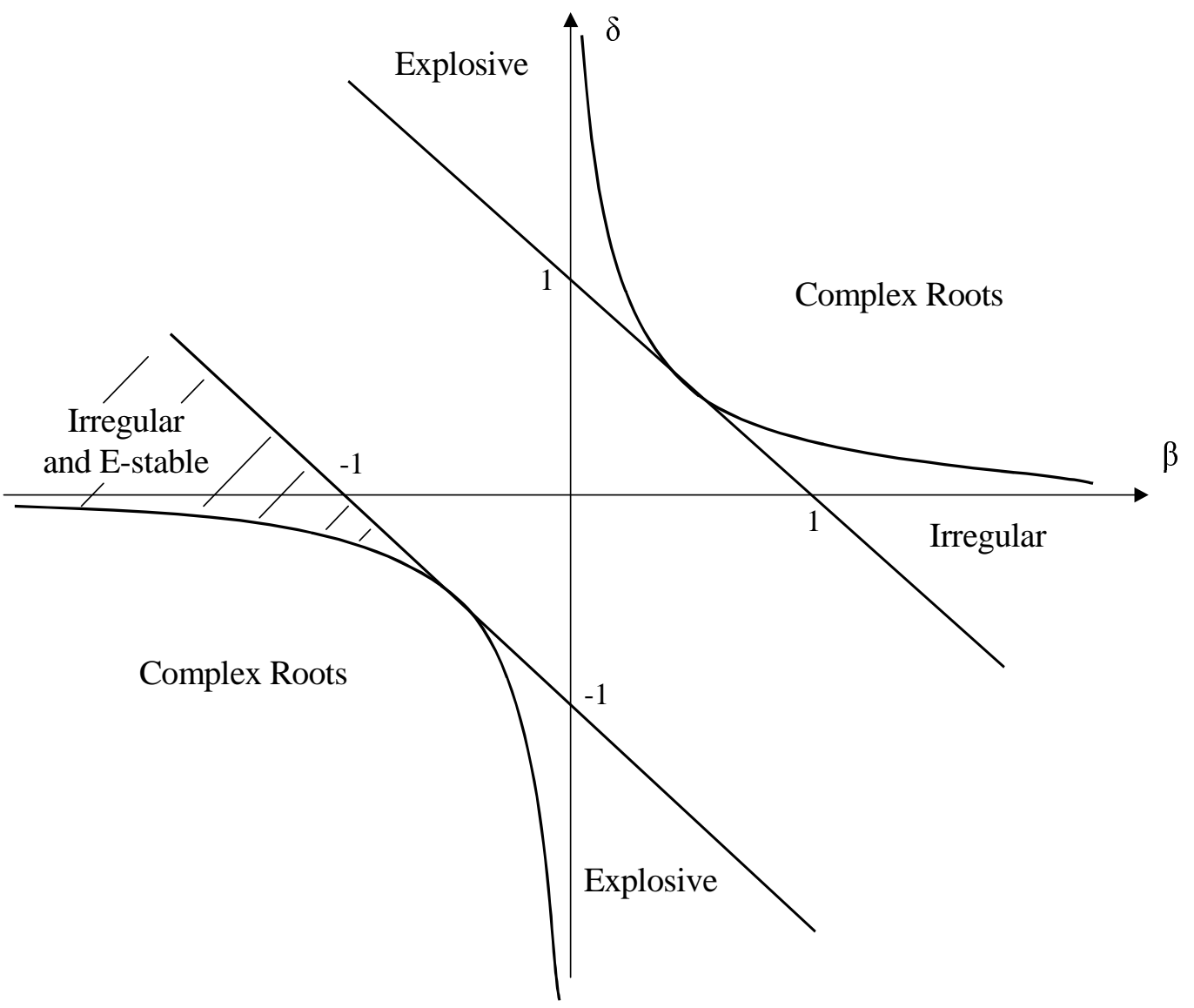

Figure 1: Region of E-stable Common Factor SSEs 


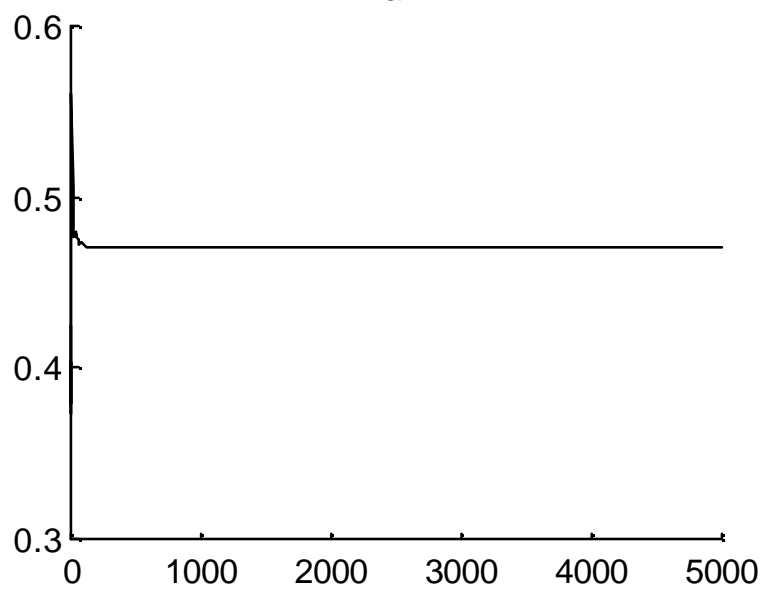

k

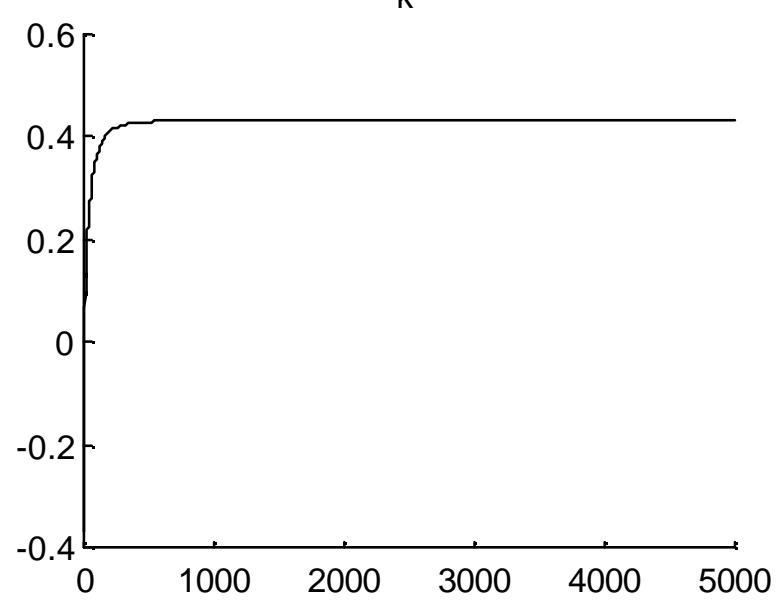

b

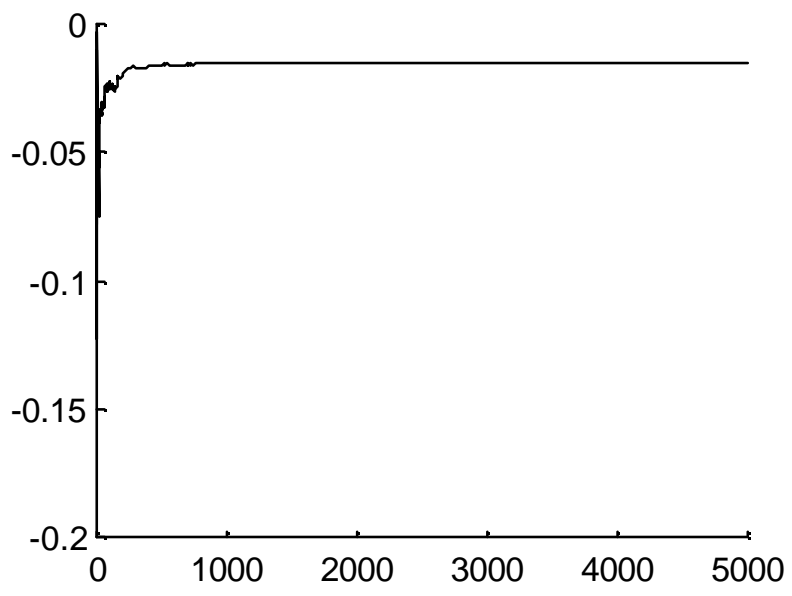

I

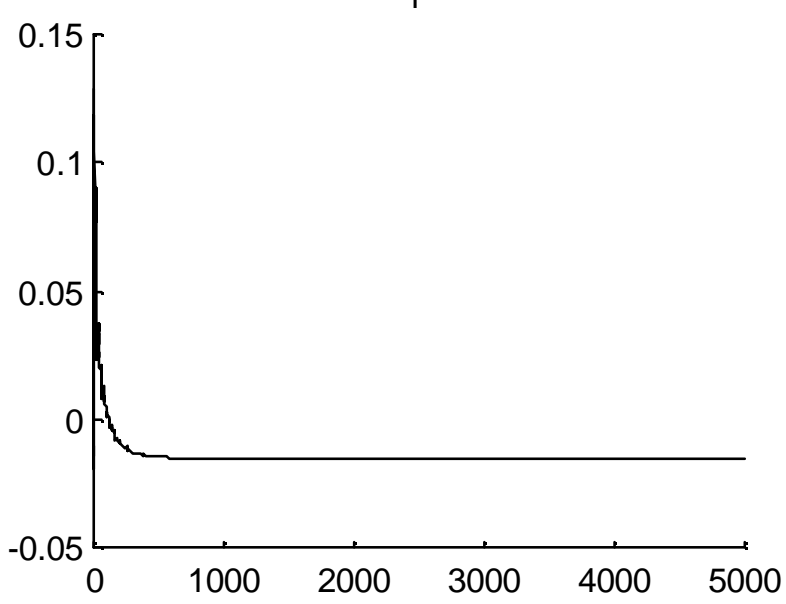

Figure $2: \beta=-3, \delta=1$ 
a
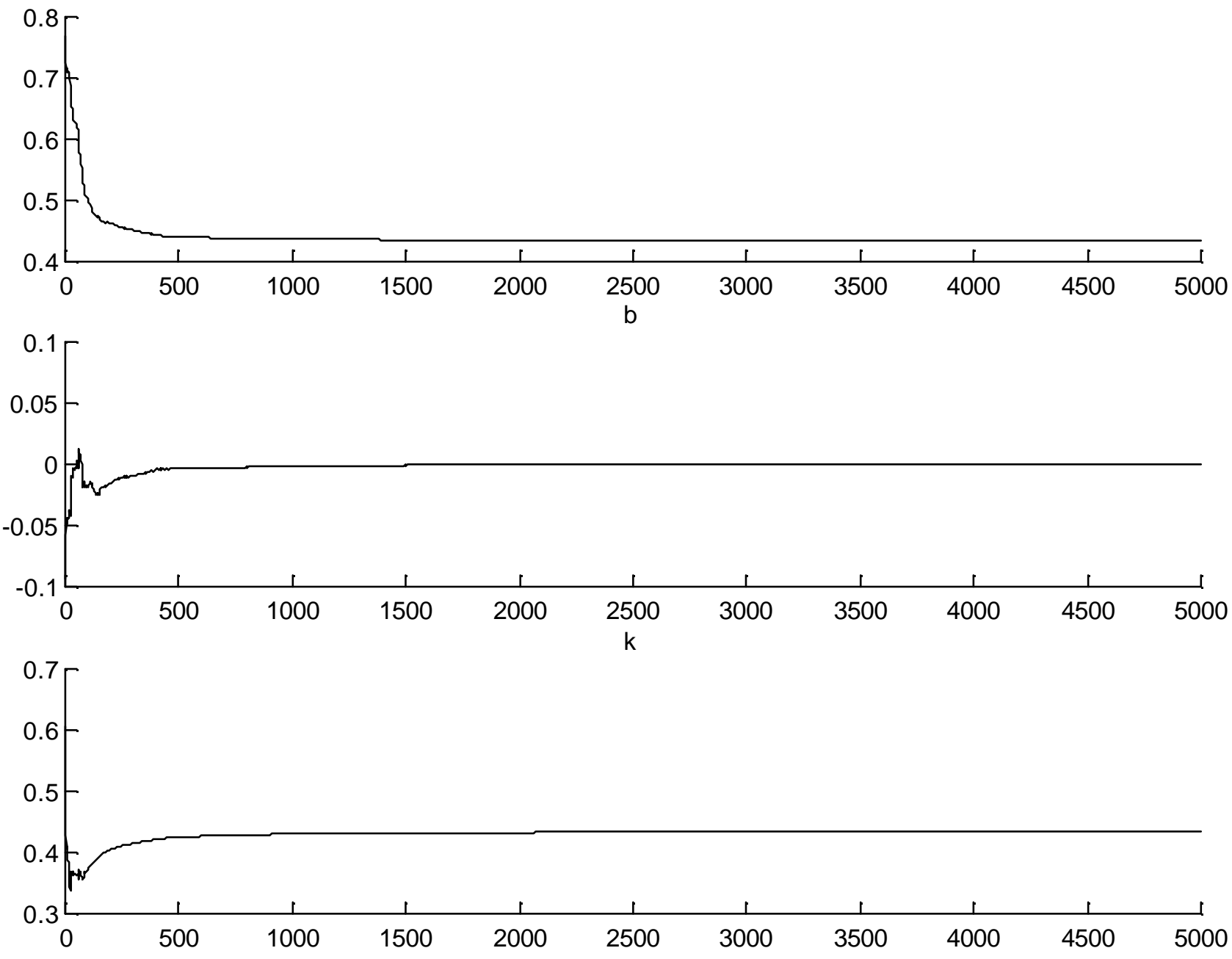

Figure 3: $\beta=-3, \delta=1$ 
a
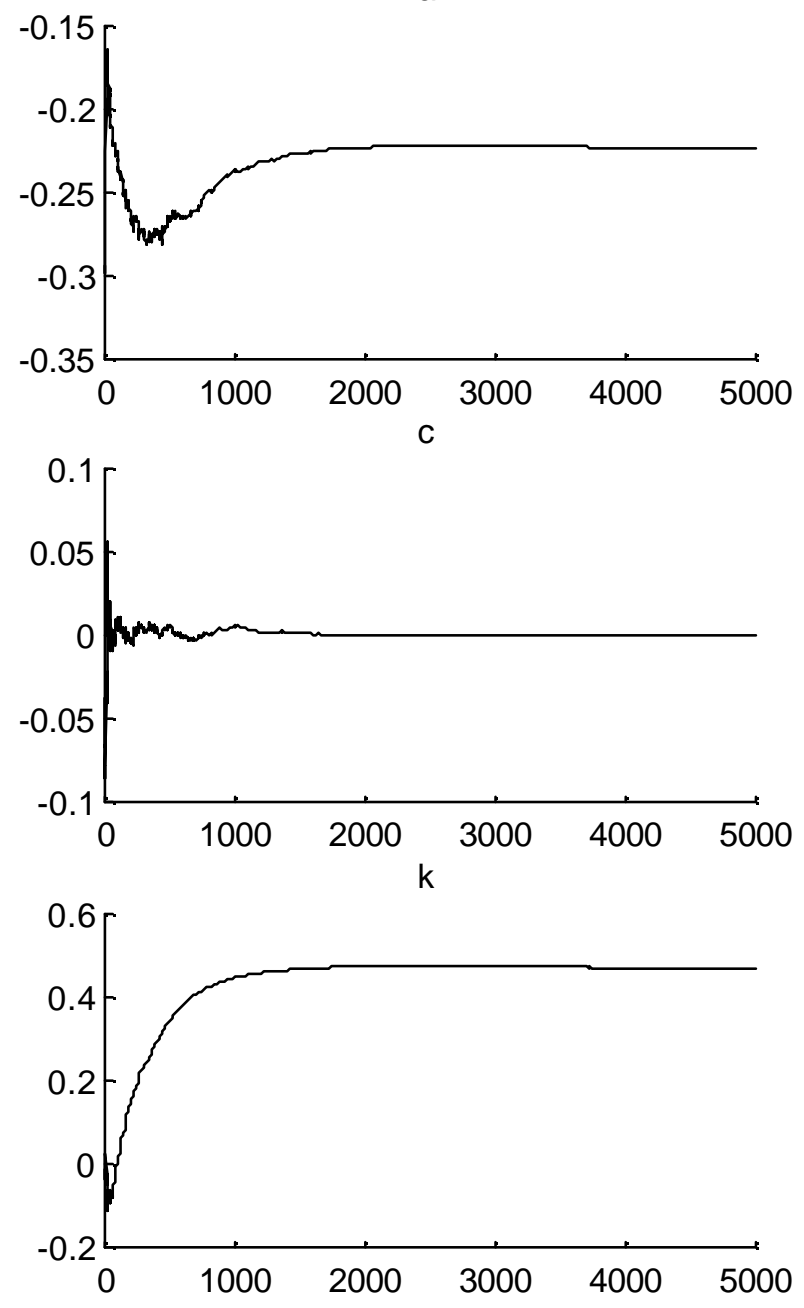

b
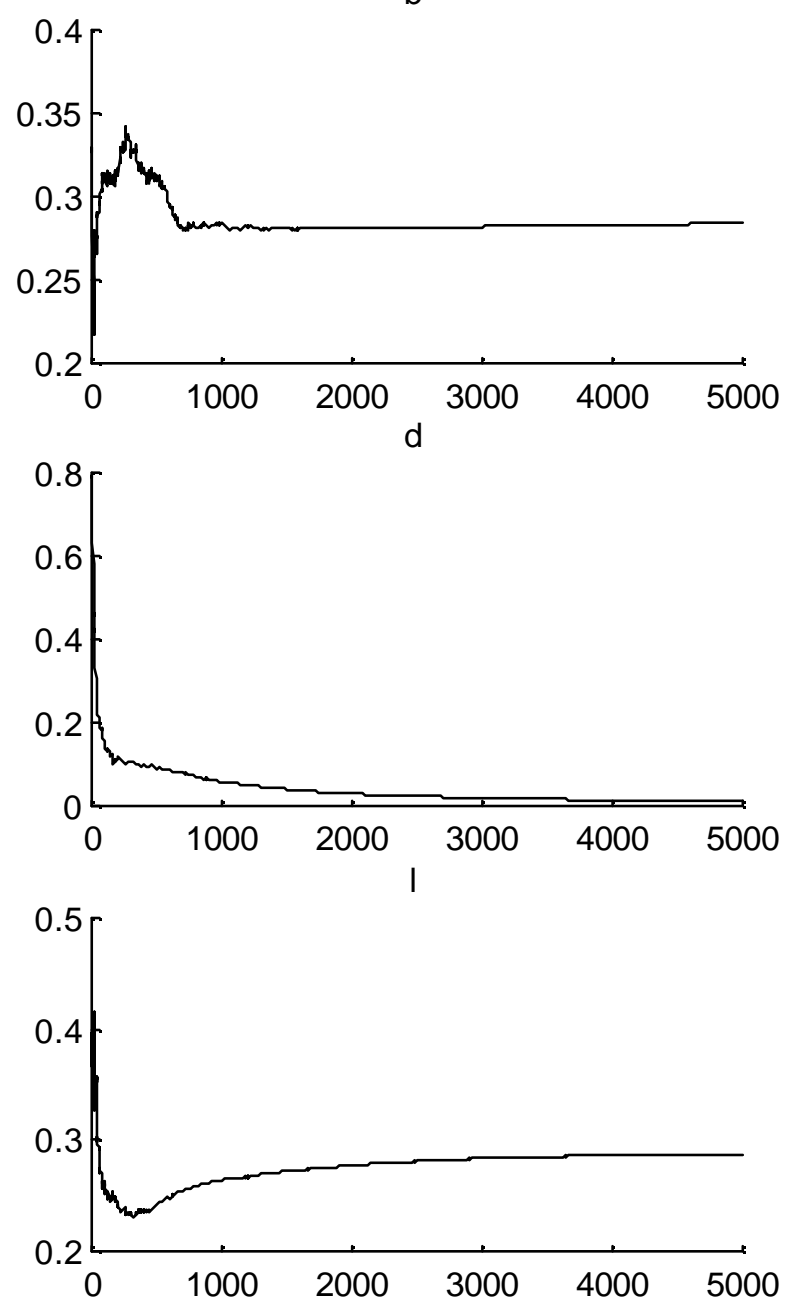

Figure $4: \beta=-3, \delta=1$ 
a
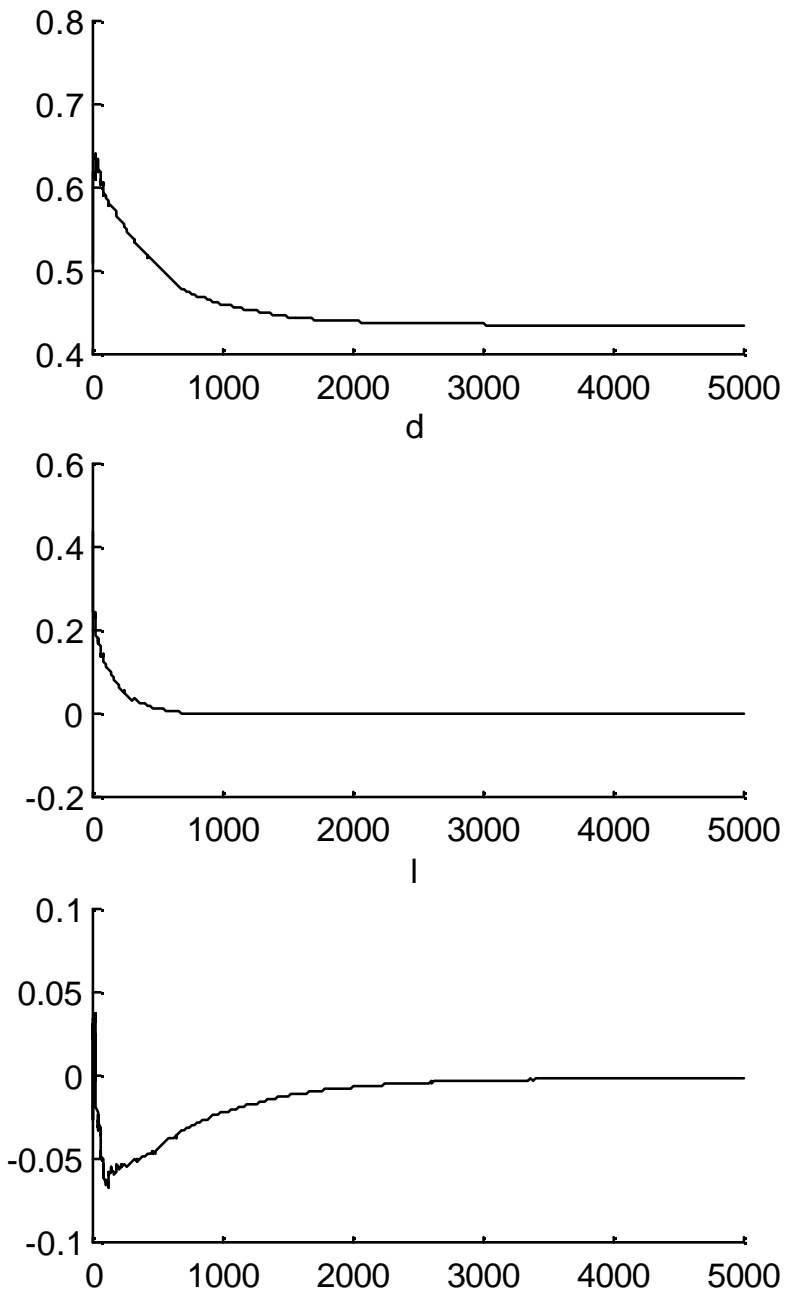

C
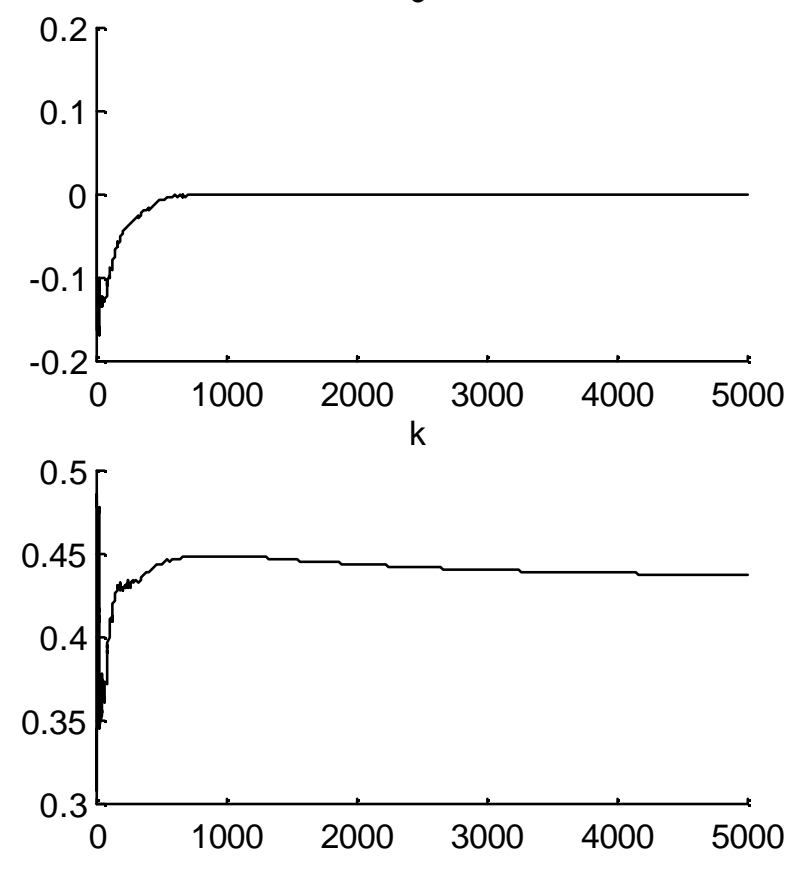

Figure $5: \beta=-3, \delta=1, \lambda=.5$ 
a
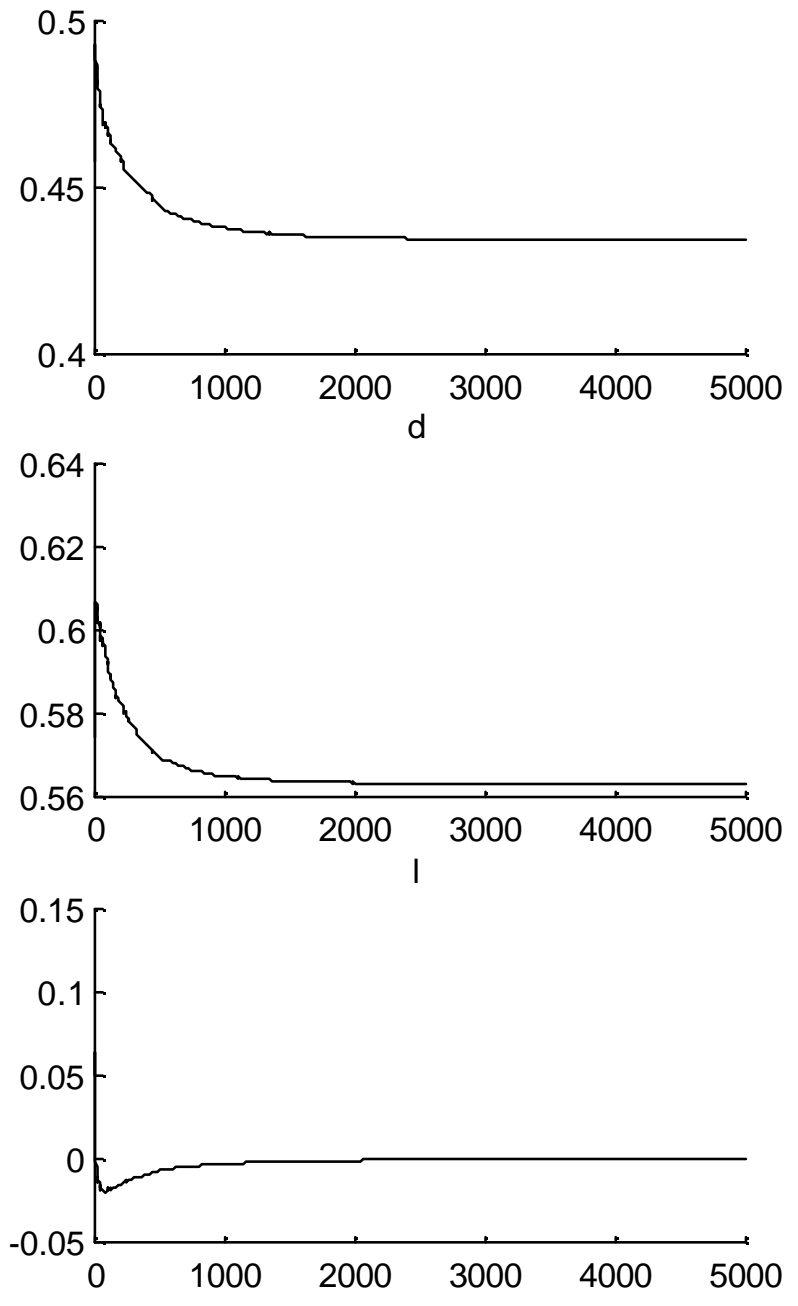

C
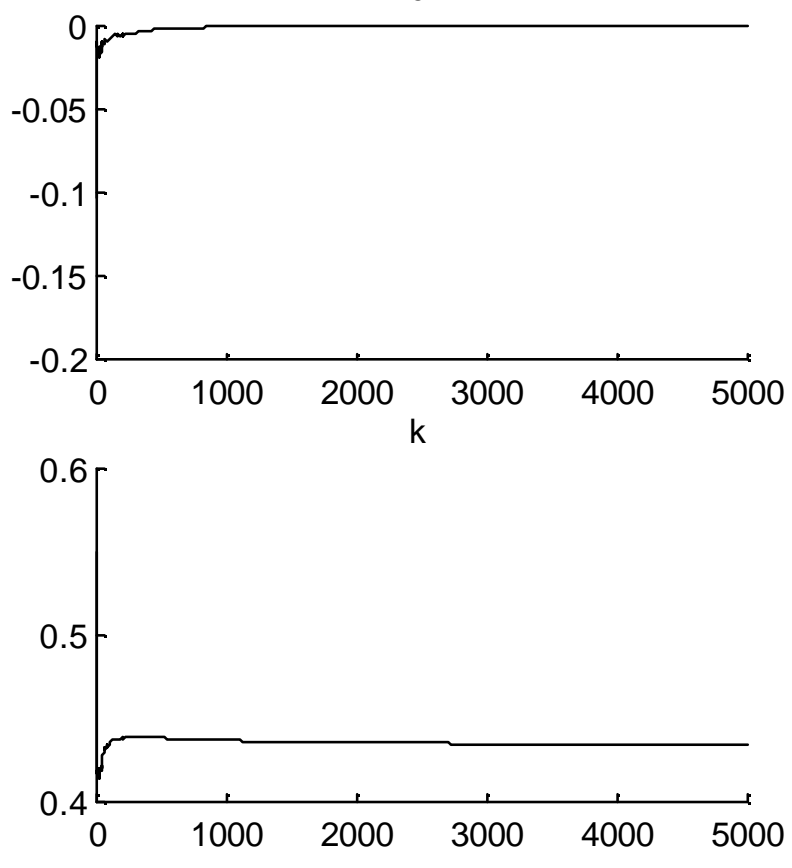

Figure $6: \beta=-3, \delta=1, \lambda=a_{2}$ 